\title{
Two-dimensional two-component plasma with adsorbing impurities
}

\author{
Alejandro Ferrero* and Gabriel Téllez ${ }^{\dagger}$ \\ Departamento de Física \\ Universidad de los Andes \\ A.A. 4976, Bogotá, Colombia
}

\begin{abstract}
We study the behavior of the two-dimensional two-component plasma in the presence of some adsorbing impurities. Using a solvable model, we find analytic expressions for the thermodynamic properties of the plasma such as the $n$-body densities, the grand potential, and the pressure. We specialize in the case where there are one or two adsorbing point impurities in the plasma, and in the case where there are one or two parallel adsorbing lines. In the former case we study the effective interaction between the impurities, due to the charge redistribution around them. The latter case is a model for electrodes with adsorbing sticky sites on their surface.
\end{abstract}

Key words: Coulomb gas; adsorption sites

\section{Introduction}

A two-component plasma (TCP) is a system composed of two different types of particles with charges $\pm e$ interacting through a Coulomb potential. In this work, we study a two-dimensional model which is exactly solvable. The solution of Poisson equation shows that in two dimensions, the Coulomb interaction between two particles, with charges $q_{1}, q_{2}$, at a distance $r$ from each other, is

$$
v(r)=-q_{1} q_{2} \ln (r / L),
$$

where $L$ is an irrelevant length scale fixing the potential reference.

Let us define the dimensionless coupling constant $\Gamma=\beta e^{2}$, where $\beta=$ $\left(k_{B} T\right)^{-1}, T$ the temperature and $k_{B}$ the Boltzmann constant. The coupling constant $\Gamma$ is the ratio between the electrostatic and thermal energy of the particles of the gas. If $\Gamma<2$, the thermal energy is high enough to prevent the collapse of particles of different sign. If $\Gamma \geq 2$, the system is unstable

\footnotetext{
*e-mail:al-ferre@uniandes.edu.co

†e-mail:gtellez@uniandes.edu.co
} 
against the collapse and the point-particles must be replaced by hard disks of diameter $a$. Using the analogy between the two-component plasma and the sineGordon quantum field theory, exact results for the thermodynamic properties of the Coulomb gas, in the bulk [2] and near a planar interface [3, 4], have been obtained when $\Gamma<2$. For $\Gamma=2$, the classical two-dimensional two-component plasma is equivalent to a quantum free Fermi gas [1]. This fact allows us to find analytic expressions for the thermodynamic properties and correlation functions of the plasma, for a wider variety of geometries $[1,5,6]$, and even in the presence of external fields [7]. In this work, we restrict our analysis to the case $\Gamma=2$.

Assuming $\Gamma=2$, we pretend to find analytical solutions for the thermodynamics of the two-component plasma in the presence of some impurities. Results have been obtained for a similar problem, the one-component plasma with adsorbing impurities $[8,9]$. For the two-component plasma, some similar problems to the present one, with adsorbing boundaries, were considered in Refs. [10, 11].

An impurity in our model can be understood as a particle, different from the charged particles of the gas. It interacts with the particles of the gas through a non-electrical potential $U_{ \pm}(\mathbf{r})$. As usual, for inhomogeneous systems under the action of an external field derived from a potential $U_{ \pm}(\mathbf{r})$, we can define a position depend fugacity due to the external potential created by the impurity $m_{ \pm}(\mathbf{r})=m e^{-\beta U_{ \pm}(\mathbf{r})}$, where $m$ is the bulk fugacity. Here we are interested in adsorbing impurities, which can be modeled with a position depend fugacity $m_{ \pm}(\mathbf{r})=m\left[1+\alpha_{ \pm} \delta(\mathbf{r}-\mathbf{R})\right]$, where $\alpha_{ \pm}$is the magnitude of the interaction of the impurity with the positive and the negative particles of the plasma (it will be called adhesivity), while $\mathbf{R}$ is the position of the impurity in the plasma. In order to prevent the collapse of the opposite charged particles, we will consider that the impurity only attracts one type of particles of the system. For instance, if the impurity only attracts the positive particles of the system, $\alpha_{+}=\alpha$ and $\alpha_{-}=0$.

This simplified model could be applied to some systems. An example is a salt solution with positive and negative ions and porous sites, which can attract some of the ions, with different sizes for each species. The size of the porous sites can be such that they are small enough in order to avoid that the larger ions get adsorbed into the sites, while the smaller ones are allowed to be adsorbed. The same salt solution may have some "dusty-points"; the contact interaction may be generated by a chemical interaction among those points and the charged particles. An example of a chemical interaction can be presented in some atoms whose valence layer is not completely filled and they can receive electrons from an atom with a small electronegativity.

The present document is organized as follows. In section 2, we briefly review the general mathematical treatment which allow us to find the thermodynamic properties and correlation functions of the two-component plasma in the presence of any external potential when $\Gamma=2[1]$. In section 3 , we consider the case where there are a few point impurities in the plasma. We concentrate specifically in the case of one or two impurities. We compute the density profiles around the impurities and the two-body correlation functions using the method of Ref. [1]. Also, we extend the treatment presented in Ref. [8] for the one- 
component plasma, to find general expressions for the grand potential, and the one- and two-body density functions for the two-component plasma with impurities, in terms of the same quantities for the unperturbed system. In section 4 , we study the two-component plasma with one or two lines of adsorbing impurities. We compute the one- and two-density functions and the grand potential using the method of Ref. [1]. A summary of the main results is presented in section 5 .

\section{Method of solution}

To solve our specific model, we use the method introduced by Cornu and Jancovici [1]. This mathematical treatment is valid for a globally neutral twocomponent Coulomb gas with a coupling constant $\Gamma=2$. Since the model is a two dimensional one, it is convenient to express the position $\mathbf{r}=(x, y)$ of the particles in complex coordinates as $z=x+i y$. As explained earlier, at $\Gamma=2$, it is mandatory to introduce a cutoff distance $a$ between particles to avoid the collapse between particles of opposite sign. This cutoff can be understood as a small diameter of the particles. It is shown in Ref. [1] that, in the limit $a \rightarrow 0$, the grand partition function $\Xi$ in this scheme is given by

$$
\ln \Xi=\operatorname{Tr}\left[\ln \left(\not \partial+m_{+}(\mathbf{r}) \frac{1+\sigma_{z}}{2}+m_{-}(\mathbf{r}) \frac{1-\sigma_{z}}{2}\right) \not \partial^{-1}\right],
$$

where $\not \partial=\sigma_{x} \partial_{x}+\sigma_{y} \partial_{y}$ is the two dimensional Dirac operator. The matrices $\sigma_{x}, \sigma_{y}, \sigma_{z}$ are the Pauli matrices. The position dependent fugacity, defined as $m_{ \pm}(\mathbf{r})=m e^{-\beta U_{ \pm}(\mathbf{r})}$, takes into account an external potential $U_{ \pm}(\mathbf{r})$ which can act differently on the positive or negative particles. The constant rescaled fugacity $m$ is related to the chemical potential $\mu$ by $m=2 \pi e^{\beta \mu} L / a^{2}$. It has units of inverse distance, and it is shown in Ref. [1], that $m^{-1}$ is the screening length.

The calculation of the $k$-body densities is reduced to find the set of Green functions $G_{s_{1} s_{2}}\left(\mathbf{r}_{1}, \mathbf{r}_{2}\right)$, with $s_{1}, s_{2}= \pm 1$, of the operator intervening in Eq. (2.1). They are the elements of a $2 \times 2$ matrix which satisfy the differential equations

$$
\left(\begin{array}{cc}
m_{+}\left(\mathbf{r}_{1}\right) & 2 \partial_{z} \\
2 \partial_{\bar{z}} & m_{-}\left(\mathbf{r}_{1}\right)
\end{array}\right)\left(\begin{array}{cc}
G_{++}\left(\mathbf{r}_{1}, \mathbf{r}_{2}\right) & G_{+-}\left(\mathbf{r}_{1}, \mathbf{r}_{2}\right) \\
G_{-+}\left(\mathbf{r}_{1}, \mathbf{r}_{2}\right) & G_{--}\left(\mathbf{r}_{1}, \mathbf{r}_{2}\right)
\end{array}\right)=\delta\left(\mathbf{r}_{1}-\mathbf{r}_{2}\right)
$$

The density $n_{ \pm}(\mathbf{r})$ and two-body truncated density correlation function $n_{ \pm}^{(2) T}\left(\mathbf{r}_{1}, \mathbf{r}_{2}\right)$ of the system are given by [1]

$$
\begin{aligned}
n_{s}(\mathbf{r}) & =m_{s}(\mathbf{r}) G_{s s}(\mathbf{r}, \mathbf{r}) \\
n_{s_{1} s_{2}}^{(2) T}\left(\mathbf{r}_{1}, \mathbf{r}_{2}\right) & =-m_{s_{1}}(\mathbf{r}) m_{s_{2}}\left(\mathbf{r}_{2}\right) G_{s_{1} s_{2}}\left(\mathbf{r}_{1}, \mathbf{r}_{2}\right) G_{s_{2} s_{1}}\left(\mathbf{r}_{2}, \mathbf{r}_{1}\right),
\end{aligned}
$$

where $T$ means truncated. More generally, the truncated $k$-body densities are 
given by [1]

$$
\begin{aligned}
n_{s_{1} \ldots s_{k}}^{(k) T}\left(\mathbf{r}_{1}, \ldots, \mathbf{r}_{k}\right)= & (-1)^{k} m_{s_{1}}\left(\mathbf{r}_{1}\right) \ldots m_{s_{k}}\left(\mathbf{r}_{k}\right) \\
& \times \sum_{\text {cycles }\left(i_{1} \cdots i_{k}\right)} G_{s_{i_{1}} s_{i_{2}}}\left(\mathbf{r}_{i_{1}}, \mathbf{r}_{i_{2}}\right) \cdots G_{s_{i_{k}} s_{i_{1}}}\left(\mathbf{r}_{i_{k}}, \mathbf{r}_{i_{1}}\right)
\end{aligned}
$$

The Green functions satisfy the useful relations

$$
\begin{aligned}
G_{s s}\left(\mathbf{r}_{1}, \mathbf{r}_{2}\right) & =\overline{G_{s s}\left(\mathbf{r}_{2}, \mathbf{r}_{1}\right)} \\
G_{s-s}\left(\mathbf{r}_{1}, \mathbf{r}_{2}\right) & =-\overline{G_{-s s}\left(\mathbf{r}_{2}, \mathbf{r}_{1}\right)}
\end{aligned}
$$

The grand potential $\beta \Omega=-\ln \Xi$ can be written as

$$
\beta \Omega=-\sum_{k} \ln \left(1+\lambda_{k}\right)
$$

where $\lambda_{k}$ are the eigenvalues of the system

$$
\begin{aligned}
& {\left[\not \partial-\frac{1}{\lambda}\left(\begin{array}{cc}
m_{+}(\mathbf{r}) & 0 \\
0 & m_{-}(\mathbf{r})
\end{array}\right)\right] \psi(\mathbf{r})=0, \text { with }} \\
& \psi(\mathbf{r})=\left(\begin{array}{c}
g(\mathbf{r}) \\
f(\mathbf{r})
\end{array}\right)
\end{aligned}
$$

a two-component spinor. Equation (2.6) shows the mentioned equivalence between the two component plasma at $\Gamma=2$ and a free fermion gas. The pressure $p$ is given as usual by

$$
\beta p=\frac{\partial \ln \Xi}{\partial A}=-\frac{1}{A} \beta \Omega,
$$

in the thermodynamic limit, where $\Omega$ is the grand potential. The "volume" of the gas is replaced by its area $A$ because we are dealing with a two-dimensional model.

The above results are valid in the continuum limit $a \rightarrow 0$. However, in this limit the partition function and other thermodynamical quantities such as the pressure and the density are divergent. We shall compute the (divergent) dominant term as a function of the cutoff $a$. On the other hand, the truncated correlation functions are finite in the limit $a \rightarrow 0$.

\section{The plasma with some point adsorbing impu- rities}

In this section, we study the two-component plasma in the presence of one or two impurities. First, we find the Green functions associated to this system at $\Gamma=2$. This allows us to find the $k$-body densities of the plasma. We will distinguish two kind of impurities. They will be called "positive" impurities when they 
attract the positive particles of the plasma, and "negative" impurities in the opposite case. This is a small abuse of language, since the impurities do not carry any electrical charge by themselves alone.

Then we shall extend the general theory presented in Ref. [8], to find general expressions for the thermodynamic properties of the system with impurities in term of those same quantities for the unperturbed system. These last results are valid for any value of the coupling constant $\Gamma$.

\subsection{Density and correlations at $\Gamma=2$}

\subsubsection{A positive impurity}

We consider a single impurity in the plasma that only adsorbs the positive charged particles. In this case the fugacity becomes $m_{+}(\mathbf{r})=m\left[1+\alpha_{1} \delta\left(\mathbf{r}-\mathbf{R}_{1}\right)\right]$ and $m_{-}(\mathbf{r})=m$. The constants $\alpha_{1}$ and $\mathbf{R}_{1}$ represent the adhesivity of the impurity and its position.

First we compute the Green functions that are needed to find the density and correlations. To solve the system (2.2) for the Green functions, we assume solutions of the form $G_{s_{1} s_{2}}=G_{s_{1} s_{2}}^{0}+G_{s_{1} s_{2}}^{1}$, where $G_{s_{1} s_{2}}^{0}$ are the bulk solutions (solutions for an unperturbed plasma with $m_{ \pm}(\mathbf{r})=m$ ) [1]

$$
\begin{aligned}
G_{ \pm \pm}^{0}\left(\mathbf{r}_{1}, \mathbf{r}_{2}\right) & =\frac{m}{2 \pi} K_{0}\left(m\left|\mathbf{r}_{1}-\mathbf{r}_{2}\right|\right) \\
G_{-+}^{0}\left(\mathbf{r}_{1}, \mathbf{r}_{2}\right) & =\frac{m}{2 \pi} \frac{\left(x_{1}-x_{2}\right)+i\left(y_{1}-y_{2}\right)}{\left|\mathbf{r}_{1}-\mathbf{r}_{2}\right|} K_{1}\left(m\left|\mathbf{r}_{1}-\mathbf{r}_{2}\right|\right) \\
& =\frac{m}{2 \pi} e^{i \theta_{12}} K_{1}\left(m\left|\mathbf{r}_{1}-\mathbf{r}_{2}\right|\right)
\end{aligned}
$$

where $\theta_{12}$ is the polar angle of the vector $\mathbf{r}_{1}-\mathbf{r}_{2}$ and $K_{0}(x), K_{1}(x)$ are the modified Bessel functions of second kind of order 0 and 1 . The bulk solutions satisfy the appropriate boundary condition at $\mathbf{r}_{1}=\mathbf{r}_{2}$ imposed by the $\delta\left(\mathbf{r}_{1}-\mathbf{r}_{2}\right)$ in Eq. (2.2). The functions $G_{s_{1} s_{2}}^{1}$ take into account the contribution of the impurity. They satisfy the differential equations

$$
\begin{aligned}
m G_{++}^{1}\left(\mathbf{r}_{1}, \mathbf{r}_{2}\right)+2 \partial_{z_{1}} G_{-+}^{1}\left(\mathbf{r}_{1}, \mathbf{r}_{2}\right) & \\
+m \alpha_{1} \delta\left(\mathbf{r}_{1}-\mathbf{R}_{1}\right)\left[G_{++}^{0}\left(\mathbf{r}_{1}, \mathbf{r}_{2}\right)+G_{++}^{1}\left(\mathbf{r}_{1}, \mathbf{r}_{2}\right)\right] & =0 \\
2 \partial_{\bar{z}_{1}} G_{++}^{1}\left(\mathbf{r}_{1}, \mathbf{r}_{2}\right)+m G_{-+}^{1}\left(\mathbf{r}_{1}, \mathbf{r}_{2}\right) & =0 \\
m G_{+-}^{1}\left(\mathbf{r}_{1}, \mathbf{r}_{2}\right)+2 \partial_{z_{1}} G_{--}\left(\mathbf{r}_{1}, \mathbf{r}_{2}\right) & \\
+m \alpha_{1} \delta\left(\mathbf{r}_{1}-\mathbf{R}_{1}\right)\left[G_{+-}^{0}\left(\mathbf{r}_{1}, \mathbf{r}_{2}\right)+G_{+-}^{1}\left(\mathbf{r}_{1}, \mathbf{r}_{2}\right)\right] & =0 \\
2 \partial_{\bar{z}_{1}} G_{+-}^{1}\left(\mathbf{r}_{1}, \mathbf{r}_{2}\right)+m G_{--}^{1}\left(\mathbf{r}_{1}, \mathbf{r}_{2}\right) & =0 .
\end{aligned}
$$

We will first solve the equations for $G_{++}^{1}$ and $G_{-+}^{1}$. We divide the space into two regions: $r_{1}<R_{1}$ and $r_{1}>R_{1}$. These regions will be denoted by the 
superscripts (1) and (2) respectively. The general solutions for $G_{ \pm+}^{1}$ are

$$
\begin{aligned}
& G_{++}^{1}\left(\mathbf{r}_{1}, \mathbf{r}_{2}\right)= \begin{cases}\sum_{l \in \mathbb{Z}} e^{i l \theta_{1}} B_{l}^{(1)} I_{l}\left(m r_{1}\right), & r_{1}<R_{1} \\
\sum_{l \in \mathbb{Z}} e^{i l \theta_{1}} A_{l}^{(2)} K_{l}\left(m r_{1}\right), & r_{1}>R_{1}\end{cases} \\
& G_{-+}^{1}\left(\mathbf{r}_{1}, \mathbf{r}_{2}\right)= \begin{cases}-\sum_{l \in \mathbb{Z}} e^{i l \theta_{1}} B_{l-1}^{(1)} I_{l}\left(m r_{1}\right), & r_{1}<R_{1} \\
\sum_{l \in \mathbb{Z}} e^{i l \theta_{1}} A_{l-1}^{(2)} K_{l}\left(m r_{1}\right), & r_{1}>R_{1} .\end{cases}
\end{aligned}
$$

with $I_{l}(x)$ and $K_{l}(x)$ the modified Bessel functions of first and second kind of order $l$. From Eq. (3.2a), we notice that $G_{++}^{1}$ is continuous, but that $G_{-+}^{1}$ must be discontinuous at $\mathbf{r}_{1}=\mathbf{R}_{1}$, due to the $\delta\left(\mathbf{r}_{1}-\mathbf{R}_{1}\right)$ term. Multiplying Eq. (3.2a) by $e^{-i l \theta_{1}}$ and integrating on $\mathbf{r}_{1}$ in a small annulus domain, centered at the origin and containing $\mathbf{R}_{1}$, gives

$$
\begin{gathered}
\left.G_{++}^{1}\left(\mathbf{r}_{1}, \mathbf{r}_{2}\right)\right|_{r_{1}=R_{1}^{+}}=\left.G_{++}^{1}\left(\mathbf{r}_{1}, \mathbf{r}_{2}\right)\right|_{r_{1}=R_{1}^{-}} \\
\frac{m \alpha_{1}}{2 \pi R_{1}}\left[G_{++}^{0}\left(\mathbf{R}_{1}, \mathbf{r}_{2}\right)+G_{++}^{1}\left(\mathbf{R}_{1}, \mathbf{r}_{2}\right)\right]= \\
-\left[\left.G_{-+}^{(1) l+1}\left(\mathbf{r}_{1}, \mathbf{r}_{2}\right)\right|_{r_{1}=R_{1}^{+}}-\left.G_{-+}^{(1) l+1}\left(\mathbf{r}_{1}, \mathbf{r}_{2}\right)\right|_{r_{1}=R_{1}^{-}}\right]
\end{gathered}
$$

where $G_{-+}^{(1) l+1}$ refers to the $l+1$ term of the sum in Eq. (3.3b). Imposing the boundary conditions (3.4), we find

$$
A_{l}^{(2)}=B_{l}^{(1)} \frac{I_{l}\left(m R_{1}\right)}{K_{l}\left(m R_{1}\right)}
$$

and

$$
B_{l}^{(1)}=-\frac{m^{3} \alpha_{1} K_{l}\left(m R_{1}\right) e^{-i l \theta_{R_{1}}} K_{0}\left(m\left|\mathbf{R}_{1}-\mathbf{r}_{2}\right|\right)}{4 \pi^{2}\left[1+\frac{m^{2} \alpha_{1}}{2 \pi} \sum_{n \in \mathbb{Z}} K_{n}\left(m R_{1}\right) I_{n}\left(m R_{1}\right)\right]},
$$

where $\theta_{R_{1}}$ is the polar angle of the vector $\mathbf{R}_{1}$.

At this point, let us comment a few details about the bulk density, for an unperturbed system, which will be useful to interpret the denominator in Eq. (3.6). The bulk density is given by $n_{ \pm}^{0}(\mathbf{r})=m G_{ \pm \pm}^{0}(\mathbf{r}, \mathbf{r})$. Nevertheless, this expression diverges, since, for small argument, $K_{0}(x) \sim \ln (2 / x)-C$, with $C \simeq 0.5772$ the Euler constant [1]. This divergence can be avoided by replacing the point particles by hard spheres of size $a$, which represents the minimum distance between two charged particles. Then, we compute the bulk density as $n_{ \pm}^{0}=m G_{ \pm \pm}^{0}\left(\mathbf{r}_{1}, \mathbf{r}_{2}\right)$ with $\left|\mathbf{r}_{1}-\mathbf{r}_{2}\right|=a \rightarrow 0$. Thus,

$$
n_{ \pm}^{0}=n_{0}=\frac{m^{2}}{2 \pi} K_{0}\left(m\left|\mathbf{r}_{1}-\mathbf{r}_{2}\right|\right)=\frac{m^{2}}{2 \pi} K_{0}(m a) \simeq \frac{m^{2}}{2 \pi}\left[\ln \frac{2}{m a}-C\right],
$$

where we used the small-argument expansion of the Bessel function $K_{0}$. Now, let us recall the expansion $K_{0}\left(m\left|\mathbf{r}_{1}-\mathbf{r}_{2}\right|\right)=\sum_{n \in \mathbb{Z}} e^{i n\left(\theta_{1}-\theta_{2}\right)} I_{n}\left(m r_{<}\right) K_{n}\left(m r_{>}\right)$ with $r_{<}=\min \left(\mathbf{r}_{1}, \mathbf{r}_{2}\right)$ and $r_{>}=\max \left(\mathbf{r}_{1}, \mathbf{r}_{2}\right)$ [12]. With this expansion, we can 
obtain another formal (divergent) expression for the bulk density

$$
n_{ \pm}^{0}\left(\mathbf{R}_{1}\right)=n_{0}=\frac{m^{2}}{2 \pi} \sum_{n \in \mathbb{Z}} K_{n}\left(m R_{1}\right) I_{n}\left(m R_{1}\right) .
$$

Since the unperturbed system is homogeneous the bulk density is constant, $n_{ \pm}^{0}\left(\mathbf{R}_{1}\right)=n_{0}$ does not depend on $\mathbf{R}_{1}$. We notice that it is this expression that precisely appears in the denominator of Eq. (3.6). Then,

$$
B_{l}^{(1)}=-\frac{m^{3} \alpha_{1} K_{l}\left(m R_{1}\right) e^{-i l \theta_{R_{1}}} K_{0}\left(m\left|\mathbf{R}_{1}-\mathbf{r}_{2}\right|\right)}{4 \pi^{2}\left(1+\alpha_{1} n_{0}\right)} .
$$

Replacing in Eqs. (3.3a) and (3.3b),

$$
\begin{aligned}
& G_{++}^{1}\left(\mathbf{r}_{1}, \mathbf{r}_{2}\right)=-\frac{m^{3} \alpha_{1}}{4 \pi^{2}\left[1+\alpha_{1} n_{0}\right]} K_{0}\left(m\left|\mathbf{R}_{1}-\mathbf{r}_{2}\right|\right) K_{0}\left(m\left|\mathbf{r}_{1}-\mathbf{R}_{1}\right|\right) \\
& G_{-+}^{1}\left(\mathbf{r}_{1}, \mathbf{r}_{2}\right)=-\frac{m^{3} \alpha_{1} e^{i \theta_{1 R_{1}}}}{4 \pi^{2}\left[1+\alpha_{1} n_{0}\right]} K_{0}\left(m\left|\mathbf{r}_{2}-\mathbf{R}_{1}\right|\right) K_{1}\left(m\left|\mathbf{r}_{1}-\mathbf{R}_{1}\right|\right)
\end{aligned}
$$

with $\theta_{1 R_{1}}$ the polar angle of the vector $\mathbf{r}_{1}-\mathbf{R}_{1}$.

In a similar way, the other two Green functions are

$$
\begin{aligned}
& G_{--}^{1}\left(\mathbf{r}_{1}, \mathbf{r}_{2}\right)=\frac{m^{3} \alpha_{1} e^{i\left(\theta_{1 R_{1}}-\theta_{2 R_{1}}\right)}}{4 \pi^{2}\left[1+\alpha_{1} n_{0}\right]} K_{1}\left(m\left|\mathbf{r}_{1}-\mathbf{R}_{1}\right|\right) K_{1}\left(m\left|\mathbf{r}_{2}-\mathbf{R}_{1}\right|\right) \\
& G_{+-}^{1}\left(\mathbf{r}_{1}, \mathbf{r}_{2}\right)=\frac{m^{3} \alpha_{1} e^{-i \theta_{2 R_{1}}}}{4 \pi^{2}\left[1+\alpha_{1} n_{0}\right]} K_{0}\left(m\left|\mathbf{r}_{1}-\mathbf{R}_{1}\right|\right) K_{1}\left(m\left|\mathbf{R}_{1}-\mathbf{r}_{2}\right|\right)
\end{aligned}
$$

with $\theta_{2 R_{1}}$ the polar angle of the vector $\mathbf{r}_{2}-\mathbf{R}_{1}$. The one-body densities can be calculated by using Eq. (2.3a), the result is

$$
\begin{aligned}
& n_{+}(\mathbf{r})=\left[1+\alpha_{1} \delta\left(\mathbf{r}-\mathbf{R}_{1}\right)\right]\left[n_{0}-\left(\frac{m^{2}}{2 \pi}\right)^{2} \frac{\alpha_{1}\left[K_{0}\left(m\left|\mathbf{R}_{1}-\mathbf{r}\right|\right)\right]^{2}}{\left[1+\alpha_{1} n_{0}\right]}\right] \\
& n_{-}(\mathbf{r})=n_{0}+\left(\frac{m^{2}}{2 \pi}\right)^{2} \frac{\alpha_{1}\left[K_{1}\left(m\left|\mathbf{R}_{1}-\mathbf{r}\right|\right)\right]^{2}}{\left[1+\alpha_{1} n_{0}\right]}
\end{aligned}
$$
is

The number of positive and negative particles of the plasma, $N_{ \pm}=\int n_{ \pm}(\mathbf{r}) d^{2} \mathbf{r}$,

$$
N_{+}=N_{-}=n_{0} \pi R^{2}-\frac{m^{2} \alpha_{1}}{4 \pi\left[1+\alpha_{1} n_{0}\right]}+\frac{n_{0} \alpha_{1}}{1+\alpha_{1} n_{0}}
$$

where we supposed that the plasma is confined in a large disk of radius $R$.

The difference $N_{+}-N_{-}=0$. This shows that the global neutrality of the system is not changed, only the charge distribution. The amount of positive 


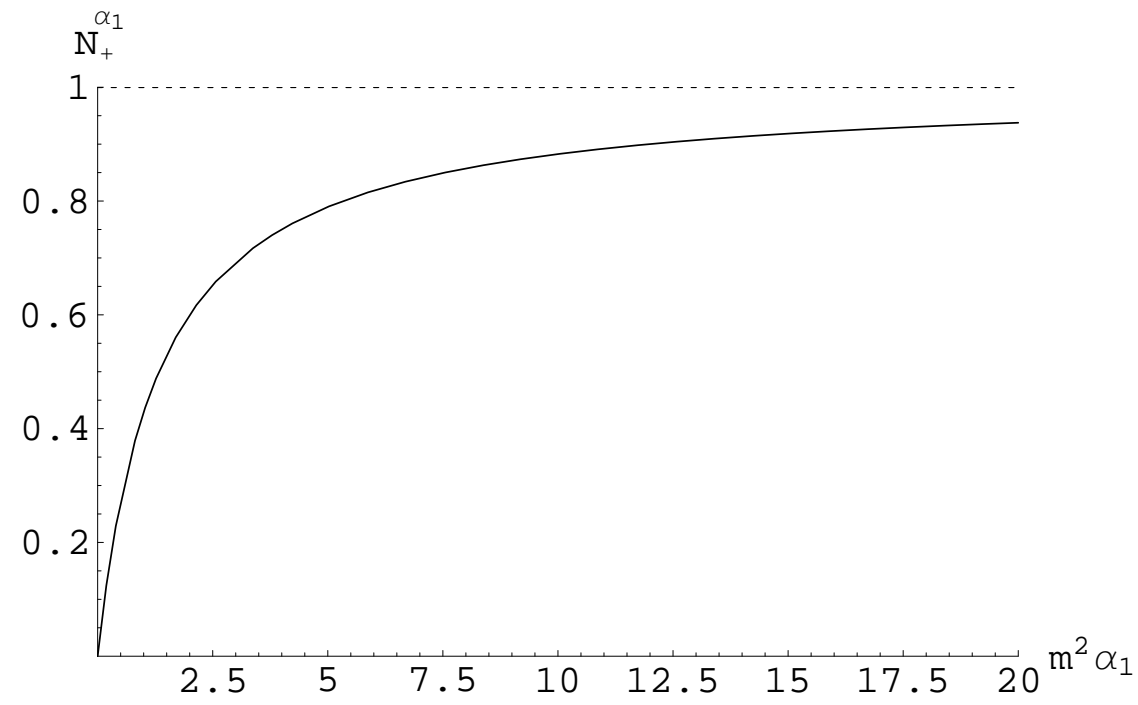

Figure 1: Average number of adsorbed particles as a function of the adhesivity. The cutoff is $m a=0.01$. The dashed line is the asymptotic value $N_{+}^{\infty}=1$ for $\alpha_{1} \rightarrow \infty$.

charge bound to the impurity, $N_{+}^{\alpha_{1}}$, is given by the term which multiply the delta distribution in Eq. (3.12a), evaluated at $\mathbf{R}_{1}$. The result is

$$
N_{+}^{\alpha_{1}}=\frac{\alpha_{1} n_{0}}{1+\alpha_{1} n_{0}},
$$

which can be interpreted as a mean occupation number or probability that the adsorbing point is occupied by a positive charge. Figure 1 shows $N_{+}^{\alpha_{1}}$ as a function of $\alpha_{1}$. As the adhesivity $\alpha_{1}$ is increased, the average number of adsorbed positive particles $N_{+}^{\alpha_{1}}$ increases, as expected. In the limit $\alpha_{1} \rightarrow \infty$, the maximum value of adsorbed particles is obtained $N_{+}^{\infty}=1$. Notice that in average there cannot be more than one adsorbed particle per impurity site. Once a particle is adsorbed, any other particle of the same sign feels a strong electrostatic repulsion that prevent it from approaching the adsorbtion site.

This localized positive adsorbed charge is the responsible of the change in the charge distribution. Since the bound charge is positive, it repels the positive particles and attracts the negative ones. As a consequence, the density of the negative particles around the impurity increases, and the density of the positive particles around the impurity decreases. As it is expected, the larger the adhesivity $\alpha_{1}$ is, the larger this effect is. This behavior can be seen in figures 2, 3 and 4 which show the density profiles of the positive and negative particles, and the charge density profile.

If the impurity attracts the negative particles, instead of the positive ones, we obtain the same results by interchanging $(+\longleftrightarrow-)$. 


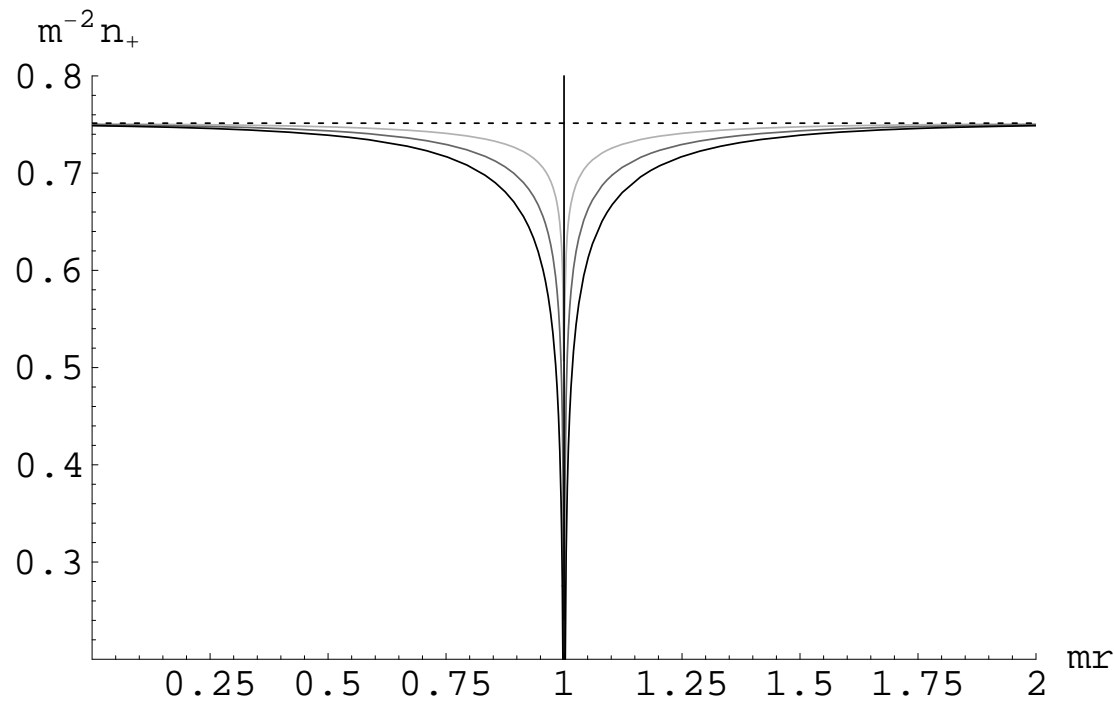

Figure 2: Density of positive particles as a function of the distance for $m^{2} \alpha_{1}=$ $1,0.5$ and 0.2 from the darkest to the lightest. The dashed line represents the bulk density. The vertical line at $m r=1$ represents the Dirac distribution related to the impurity position which is located at $m \mathbf{R}_{1}=\hat{x}$. The angular difference is $\theta-\theta_{R_{1}}=0$. The cutoff is $m a=0.01$. 


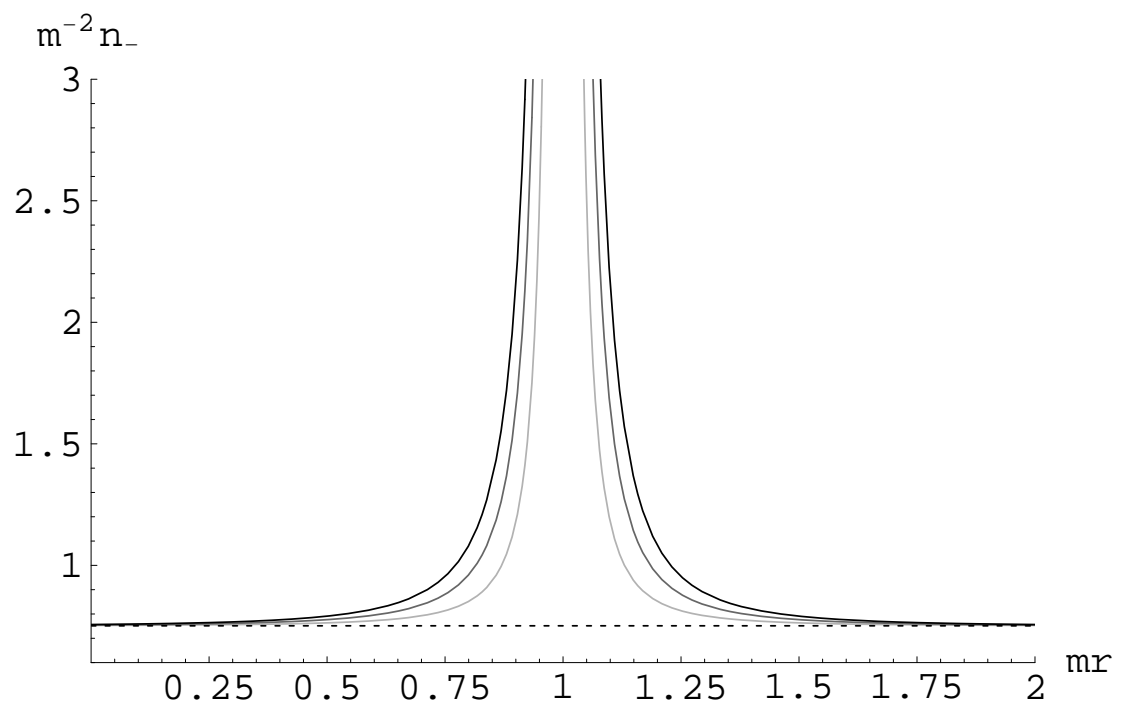

Figure 3: Density of negative particles as a function of the distance for $m^{2} \alpha_{1}=$ $1,0.5$ and 0.2 from the darkest to the lightest. The dashed line represents the bulk density. The angular difference is $\theta-\theta_{R_{1}}=0$. The cutoff is $m a=0.01$.

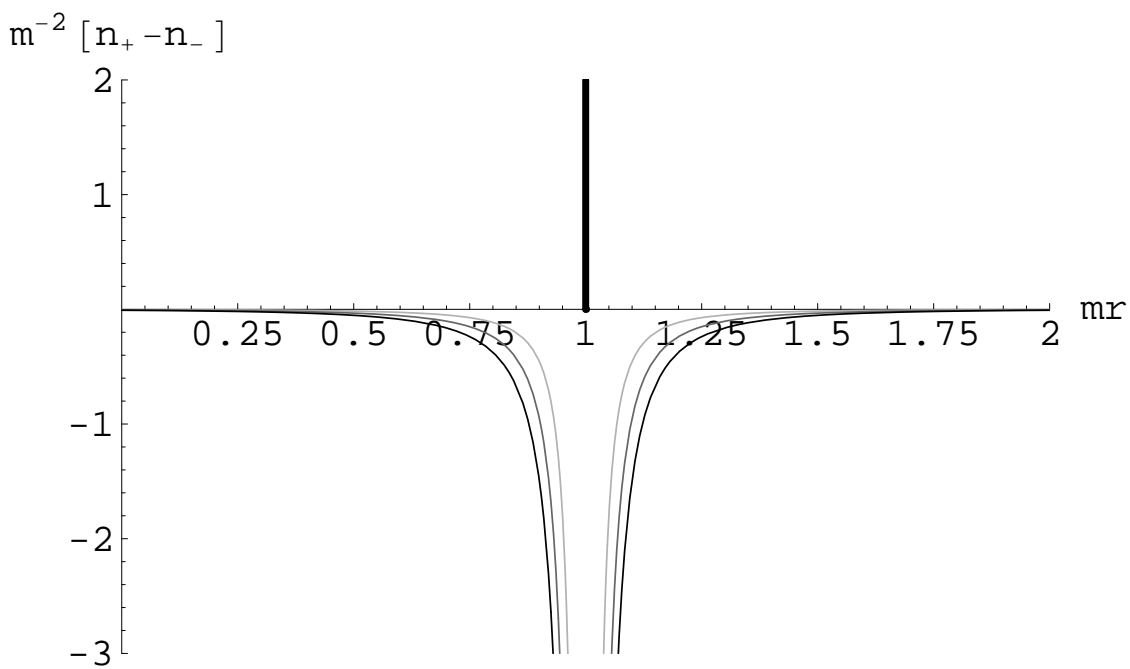

Figure 4: Charge density profile as a function of the distance for $m^{2} \alpha_{1}=1,0.5$ and 0.2 from the darkest to the lightest. The vertical line at $m r=1$ represents the Dirac distribution related to the impurity position which is located at $m \mathbf{R}_{1}=\hat{x}$. The angular difference is $\theta-\theta_{R_{1}}=0$. The cutoff is $m a=0.01$. 


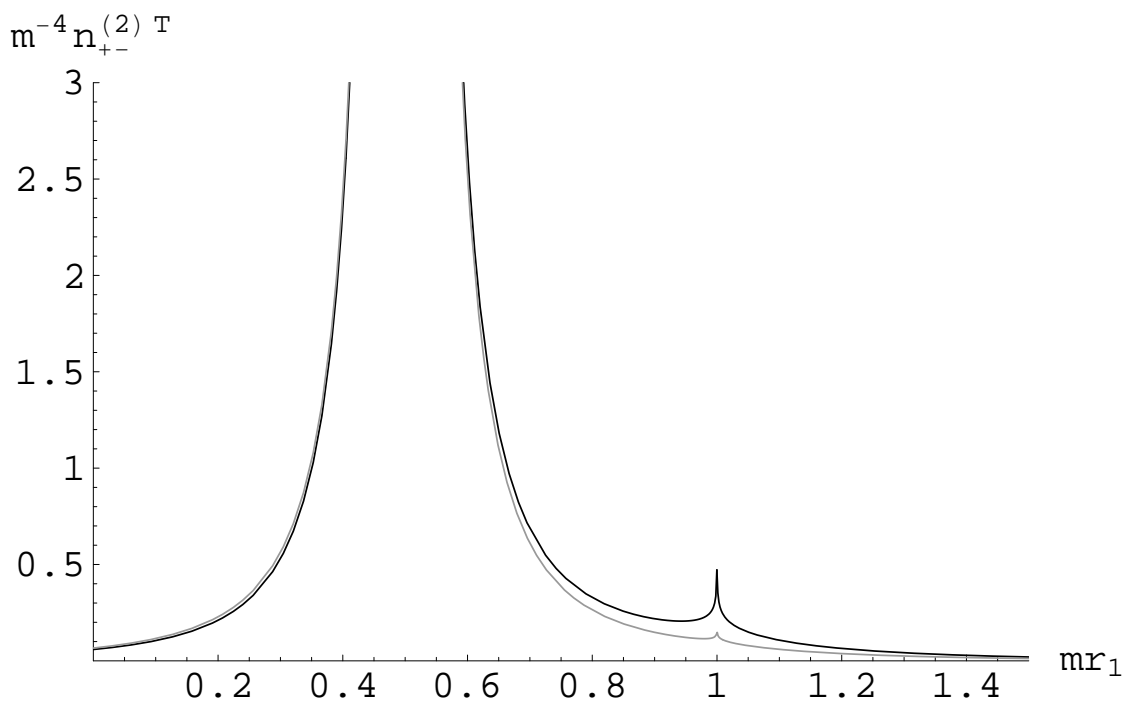

Figure 5: $n_{+-}^{(2) T}\left(\mathbf{r}_{1}, \mathbf{r}_{2}\right)$ as a function of $r_{1}$ for $m^{2} \alpha_{1}=10$ and 0.5 from the darkest to lightest. We assumed $m r_{2}=0.5$ and $m R_{1}=1$. The angular differences are $\theta_{1}-\theta_{2}=\theta_{1}-\theta_{R_{1}}=0$ : we are looking through a line which passes over $\mathbf{r}_{2}$ and $\mathbf{R}_{1}$. The delta function at $\mathbf{r}_{1}=\mathbf{R}_{1}$ is not showed.

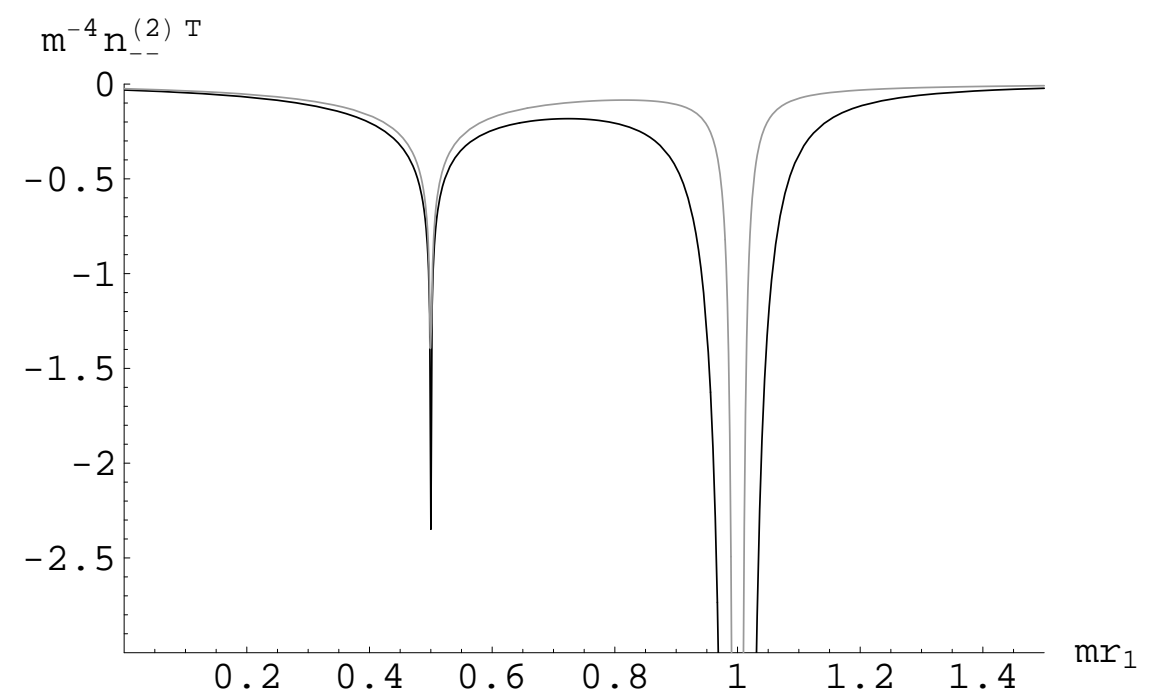

Figure 6: $n_{--}^{(2) T}\left(\mathbf{r}_{1}, \mathbf{r}_{2}\right)$ as a function of the distance. We assume the same parameter values as in figure 5 . 


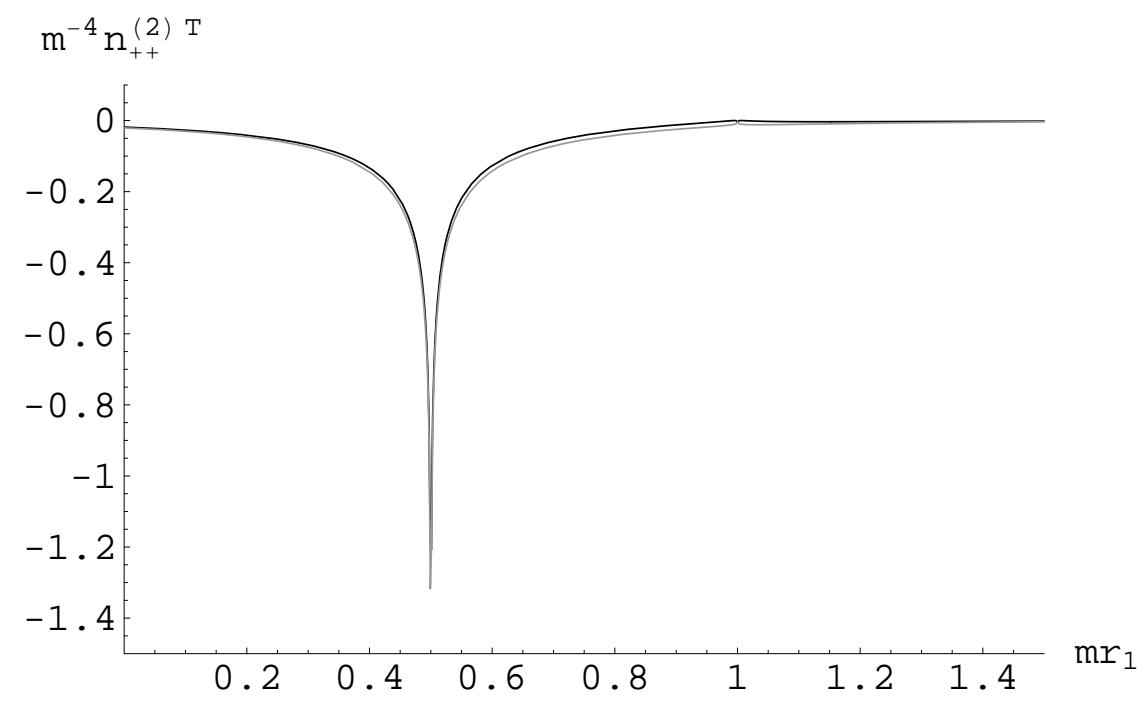

Figure $7: n_{++}^{(2) T}\left(\mathbf{r}_{1}, \mathbf{r}_{2}\right)$ as a function of the distance. We assume the same parameter values as in figure 5 . The delta function is not plotted.

As seen in figures 2, 3 and 4, a charge polarization cloud is formed around the impurity. It is interesting to evaluate the electric potential created by this charge distribution. To compute the electric potential, we use the Poisson equation $\nabla^{2} \phi(\mathbf{r})=-2 \pi e\left[n_{+}(\mathbf{r})-n_{-}(\mathbf{r})\right]$, where $n_{ \pm}(\mathbf{r})$ are given in Eqs. (3.12). In the thermodynamic limit the plasma is large enough and we can assume an angular symmetry around the impurity (locating it at the origin). Thus, Poisson equation reduces to

$$
\frac{1}{r} \frac{d}{d r}\left[r \frac{d}{d r} \phi(r)\right]=-2 \pi e\left[n_{+}(r)-n_{-}(r)\right] .
$$

Integrating, we find the electric potential

$$
\phi(r)=\frac{e m^{2} \alpha_{1}}{2 \pi\left[1+\alpha_{1} n_{0}\right]}\left[K_{0}(m r)\right]^{2}=\frac{e m^{2} N_{+}^{\alpha_{1}}}{2 n_{0} \pi}\left[K_{0}(m r)\right]^{2} .
$$

From Eq. (3.16) we can see that close to the impurity, the potential behaves as $[\ln (m r)]^{2}$, which is stronger than the bare Coulomb potential $-\ln (r / L)$. This is due to the strong electrostatic coupling considered here [17], for smaller coupling, one would expect that the electric potential close to the impurity behaves as the bare Coulomb potential. Far away from it, the interaction decays exponentially due to the screening effect.

Replacing the results for the Green functions, Eqs. (3.10) and (3.11), into Eq. (2.3b) allows us to obtain the two-body density correlation functions. Figures 5,6 , and 7 show plots of the two-body densities, for different values of the adhesivity. 


\subsubsection{Two positive impurities}

Now, we consider two impurities located at $\mathbf{R}_{1}$ and $\mathbf{R}_{2}$ with adhesivities $\alpha_{1}$ and $\alpha_{2}$ respectively, both attracting positive particles. The fugacities are now given by $m_{+}(\mathbf{r})=m\left[1+\alpha_{1} \delta\left(\mathbf{r}-\mathbf{R}_{1}\right)+\alpha_{2} \delta\left(\mathbf{r}-\mathbf{R}_{2}\right)\right]$ and $m_{-}(\mathbf{r})=m$.

To solve the system of equations (2.2) for the Green functions, we assume they are of the form $G_{s_{1} s_{2}}=G_{s_{1} s_{2}}^{0}+G_{s_{1} s_{2}}^{1}+G_{s_{1} s_{2}}^{2}$ where $G_{s_{1} s_{2}}^{1}$ are the solutions previously found for one impurity located at $\mathbf{R}_{1}$. Without lost of generality we choose $R_{2}>R_{1}$. The space is now divided into three regions. The first region is $r_{1}<R_{1}$, the second region is $R_{1}<r_{1}<R_{2}$ and the third one is $R_{2}<r_{1}$. The differential equations satisfied by $G_{ \pm+}^{2}$ are

$$
\begin{aligned}
m\left[1+\alpha_{1} \delta\left(\mathbf{r}_{1}-\mathbf{R}_{1}\right)+\alpha_{2} \delta\left(\mathbf{r}_{1}-\mathbf{R}_{2}\right)\right] G_{++}^{2}\left(\mathbf{r}_{1}, \mathbf{r}_{2}\right) & \\
+m \alpha_{2} \delta\left(\mathbf{r}_{1}-\mathbf{R}_{2}\right)\left[G_{++}^{0}\left(\mathbf{r}_{1}, \mathbf{r}_{2}\right)+G_{++}^{1}\left(\mathbf{r}_{1}, \mathbf{r}_{2}\right)\right] & \\
+2 \partial_{z_{1}} G_{-+}^{2}\left(\mathbf{r}_{1}, \mathbf{r}_{2}\right) & =0 \\
2 \partial_{\bar{z}_{1}} G_{++}^{2}\left(\mathbf{r}_{1}, \mathbf{r}_{2}\right)+m G_{-+}^{2}\left(\mathbf{r}_{1}, \mathbf{r}_{2}\right) & =0 .
\end{aligned}
$$

with general solutions of the form

$$
\begin{aligned}
G_{++}^{2}\left(\mathbf{r}_{1}, \mathbf{r}_{2}\right) & = \begin{cases}\sum_{l \in \mathbb{Z}} e^{i l \theta_{1}} B_{l}^{(1)} I_{l}\left(m r_{1}\right) & r_{1}<R_{1} \\
\sum_{l \in \mathbb{Z}} e^{i l \theta_{1}}\left[B_{l}^{(2)} I_{l}\left(m r_{1}\right)+A_{l}^{(2)} K_{l}\left(m r_{1}\right)\right] & R_{1}<r_{1}<R_{2} \\
\sum_{l \in \mathbb{Z}} e^{i l \theta_{1}} A_{l}^{(3)} K_{l}\left(m r_{1}\right) & R_{2}<r_{1}\end{cases} \\
G_{-+}^{2}\left(\mathbf{r}_{1}, \mathbf{r}_{2}\right) & = \begin{cases}-\sum_{l \in \mathbb{Z}} e^{i l \theta_{1}} B_{l-1}^{(1)} I_{l}\left(m r_{1}\right) & r_{1}<R_{2} \\
\sum_{l \in \mathbb{Z}} e^{i l \theta_{1}}\left[-B_{l-1}^{(2)} I_{l}\left(m r_{1}\right)+A_{l-1}^{(2)} K_{l}\left(m r_{1}\right)\right] & R_{1}<r_{1}<R_{2} \\
\sum_{l \in \mathbb{Z}} e^{i l \theta_{1}} A_{l-1}^{(3)} K_{l}\left(m r_{1}\right) & R_{2}<r_{1}\end{cases}
\end{aligned}
$$

The delta functions in Eq. (3.17a) impose that $G_{++}^{2}$ is continuous at $\mathbf{r}_{1}=\mathbf{R}_{1}$ and $\mathbf{r}_{1}=\mathbf{R}_{2}$ but $G_{-+}^{2}$ is discontinuous. Imposing these four boundary conditions, we find after some algebra

$$
\begin{aligned}
A_{l}^{(2)} & =-\frac{m^{4} \alpha_{1} \alpha_{2} K_{0}\left(m\left|\mathbf{R}_{1}-\mathbf{R}_{2}\right|\right)}{4 \pi^{2} \eta} I_{l}\left(m R_{1}\right) e^{-i l \theta_{R_{1}}} D_{1} \\
A_{l}^{(3)} & =\frac{m^{2} \alpha_{2}}{2 \pi \eta} I_{l}\left(m R_{2}\right) e^{-i l \theta_{R_{2}}}\left[1+\alpha_{1} n_{0}\right] D_{1}+A_{l}^{(2)} \\
B_{l}^{(2)} & =\frac{m^{2} \alpha_{2}}{2 \pi \eta} K_{l}\left(m R_{2}\right) e^{-i l \theta_{R_{2}}}\left[1+\alpha_{1} n_{0}\right] D_{1} \\
B_{l}^{(1)} & =B_{l}^{(2)}-\frac{m^{4} \alpha_{1} \alpha_{2} K_{0}\left(m\left|\mathbf{R}_{1}-\mathbf{R}_{2}\right|\right)}{4 \pi^{2} \eta} K_{l}\left(m R_{1}\right) e^{-i l \theta_{R_{1}}} D_{1}
\end{aligned}
$$


with

$$
\begin{aligned}
D_{1} & =-\frac{m}{2 \pi} K_{0}\left(m\left|\mathbf{r}_{2}-\mathbf{R}_{2}\right|\right)+\frac{m^{3} \alpha_{1} K_{0}\left(m\left|\mathbf{R}_{1}-\mathbf{r}_{2}\right|\right) K_{0}\left(m\left|\mathbf{R}_{1}-\mathbf{R}_{2}\right|\right)}{4 \pi^{2}\left[1+\alpha_{1} n_{0}\right]}, \\
\eta & =1+\alpha_{1} n_{0}+\alpha_{2} n_{0}+\alpha_{1} \alpha_{2} n_{0}^{2}-\frac{m^{4} \alpha_{1} \alpha_{2}}{4 \pi^{2}}\left[K_{0}\left(m\left|\mathbf{R}_{1}-\mathbf{R}_{2}\right|\right)\right]^{2}
\end{aligned}
$$

The functions $G_{ \pm+}^{2}$ are therefore

$$
\begin{aligned}
G_{++}^{2}\left(\mathbf{r}_{1}, \mathbf{r}_{2}\right) & =\frac{m^{2} \alpha_{2}}{2 \pi \eta} D_{1}\left[\left[1+\alpha_{1} n_{0}\right] K_{0}\left(m\left|\mathbf{r}_{1}-\mathbf{R}_{2}\right|\right)\right. \\
& \left.-\frac{m^{2} \alpha_{1}}{2 \pi} K_{0}\left(m\left|\mathbf{r}_{1}-\mathbf{R}_{1}\right|\right) K_{0}\left(m\left|\mathbf{R}_{1}-\mathbf{R}_{2}\right|\right)\right] \\
G_{-+}^{2}\left(\mathbf{r}_{1}, \mathbf{r}_{2}\right) & =\frac{m^{2} \alpha_{2}}{2 \pi \eta} D_{1}\left[\left[1+\alpha_{1} n_{0}\right] K_{1}\left(m\left|\mathbf{r}_{1}-\mathbf{R}_{2}\right|\right) e^{i \theta_{1 R_{2}}}\right. \\
& -\frac{m^{2} \alpha_{1}}{2 \pi} K_{1}\left(m\left|\mathbf{r}_{1}-\mathbf{R}_{1}\right|\right) e^{\left.i \theta_{1 R_{1}} K_{0}\left(m\left|\mathbf{R}_{1}-\mathbf{R}_{2}\right|\right)\right] .}
\end{aligned}
$$

Following some similar steps, we find the other two Green functions

$$
\begin{aligned}
& G_{--}^{2}\left(\mathbf{r}_{1}, \mathbf{r}_{2}\right)=-\frac{m^{2} \alpha_{2}}{2 \pi \eta} D_{2}\left[\left[1+\alpha_{1} n_{0}\right] K_{1}\left(m\left|\mathbf{r}_{1}-\mathbf{R}_{2}\right|\right) e^{i \theta_{1 R_{2}}}\right. \\
& \left.-\frac{m^{2} \alpha_{1}}{2 \pi} K_{1}\left(m\left|\mathbf{r}_{1}-\mathbf{R}_{1}\right|\right) e^{i \theta_{1 R_{1}}} K_{0}\left(m\left|\mathbf{R}_{1}-\mathbf{R}_{2}\right|\right)\right] \\
& G_{+-}^{2}\left(\mathbf{r}_{1}, \mathbf{r}_{2}\right)=-\frac{m^{2} \alpha_{2}}{2 \pi \eta} D_{2}\left[\left[1+\alpha_{1} n_{0}\right] K_{0}\left(m\left|\mathbf{r}_{1}-\mathbf{R}_{2}\right|\right)\right. \\
& \left.-\frac{m^{2} \alpha_{1}}{2 \pi} K_{0}\left(m\left|\mathbf{r}_{1}-\mathbf{R}_{1}\right|\right) K_{0}\left(m\left|\mathbf{R}_{1}-\mathbf{R}_{2}\right|\right)\right]
\end{aligned}
$$

with

$$
\begin{aligned}
D_{2} & =-\frac{m}{2 \pi} K_{1}\left(m\left|\mathbf{R}_{2}-\mathbf{r}_{2}\right|\right) e^{-i \theta_{2} R_{2}} \\
& +\frac{m^{3} \alpha_{1} K_{1}\left(m\left|\mathbf{R}_{1}-\mathbf{r}_{2}\right|\right) K_{0}\left(m\left|\mathbf{R}_{2}-\mathbf{R}_{1}\right|\right) e^{-i \theta_{2 R_{1}}}}{4 \pi^{2}\left[1+\alpha_{1} n_{0}\right]}
\end{aligned}
$$

Replacing these Green functions in Eq. (2.3a) gives the density profiles. Figure 8 shows the charge density profile. It can be seen that the charge density decreases around the adsorbing points like it was found in the problem with one impurity. The density depends on the adhesivity as well as of the distance between the impurities. An important effect arises when the adsorbing points are close enough which indicates the existence of an indirect interaction between the impurities (see section 3.2.3). 


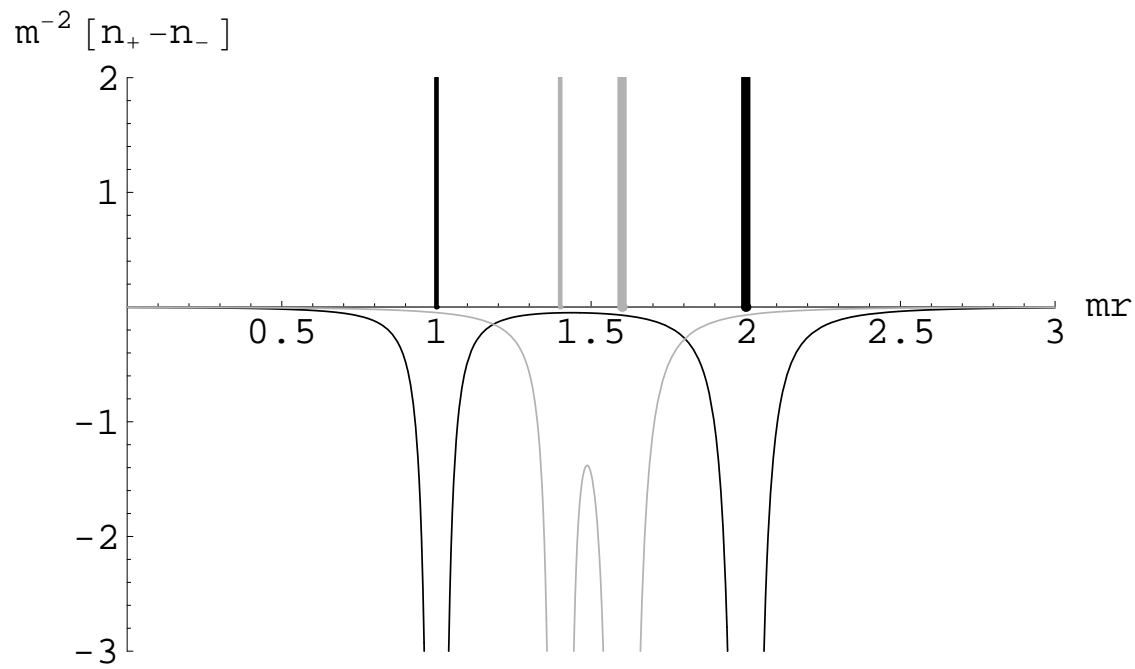

Figure 8: Charge density profile for two "positive" impurities. The adhesivities are $m^{2} \alpha_{1}=0.2$ and $m^{2} \alpha_{2}=0.5$. The positions of the impurities are on the $x$ axis at $m R_{1}=1, m R_{1}=1.4$ and $m R_{2}=2, m R_{2}=1.6$, respectively from the darkest to the lightest. All the angular differences are zero. The cutoff is $m a=0.01$.

\subsubsection{One positive and one negative impurity}

Now we consider the case when the second impurity only adsorbs the negative particles. The fugacities for this configuration are $m_{+}(\mathbf{r})=m\left[1+\alpha_{1} \delta\left(\mathbf{r}-\mathbf{R}_{1}\right)\right]$ and $m_{-}(\mathbf{r})=m\left[1+\alpha_{2} \delta\left(\mathbf{r}-\mathbf{R}_{2}\right)\right]$. Assuming a similar solution as in Eq. (3.18), and after establishing the appropriate boundary conditions for this case, we solve the linear system (2.2), to find

$$
\begin{aligned}
G_{++}\left(\mathbf{r}_{1}, \mathbf{r}_{2}\right) & =G_{++}^{0}\left(\mathbf{r}_{1}, \mathbf{r}_{2}\right)+G_{++}^{1}\left(\mathbf{r}_{1}, \mathbf{r}_{2}\right) \\
& +\frac{m^{2} \alpha_{2}}{2 \pi \bar{\eta}} D_{3}\left[\left[1+\alpha_{1} n_{0}\right] K_{1}\left(m\left|\mathbf{r}_{1}-\mathbf{R}_{2}\right|\right) e^{-i \theta_{1 R_{2}}}\right. \\
& \left.-\frac{m^{2} \alpha_{1}}{2 \pi} K_{0}\left(m\left|\mathbf{r}_{1}-\mathbf{R}_{1}\right|\right) e^{-i \theta_{R_{1} R_{2}}} K_{1}\left(m\left|\mathbf{R}_{1}-\mathbf{R}_{2}\right|\right)\right], \\
G_{-+}\left(\mathbf{r}_{1}, \mathbf{r}_{2}\right) & =G_{-+}^{0}\left(\mathbf{r}_{1}, \mathbf{r}_{2}\right)+G_{-+}^{1}\left(\mathbf{r}_{1}, \mathbf{r}_{2}\right) \\
& -\frac{m^{2} \alpha_{2}}{2 \pi \bar{\eta}} D_{3}\left[\left[1+\alpha_{1} n_{0}\right] e^{i \theta_{R_{2}} K_{0}\left(m\left|\mathbf{r}_{1}-\mathbf{R}_{2}\right|\right) e^{-i \theta_{1 R_{2}}}}\right. \\
& \left.+\frac{m^{2} \alpha_{1}}{2 \pi} K_{1}\left(m\left|\mathbf{r}_{1}-\mathbf{R}_{1}\right|\right) e^{i\left(\theta_{1 R_{1}}-\theta_{R_{1} R_{2}}\right)} K_{1}\left(m\left|\mathbf{R}_{1}-\mathbf{R}_{2}\right|\right)\right],
\end{aligned}
$$


with

$$
\begin{aligned}
D_{3} & =\frac{m^{2}}{2 \pi} e^{i \theta_{2 R_{2}}} K_{1}\left(m\left|\mathbf{R}_{2}-\mathbf{r}_{2}\right|\right) \\
& -\frac{m^{3} \alpha_{1} K_{0}\left(m\left|\mathbf{R}_{1}-\mathbf{r}_{2}\right|\right) e^{i \theta_{R_{1} R_{2}}} K_{1}\left(m\left|\mathbf{R}_{2}-\mathbf{R}_{1}\right|\right)}{4 \pi^{2}\left[1+\alpha_{1} n_{0}\right]} \\
\bar{\eta} & =1+\alpha_{1} n_{0}+\alpha_{2} n_{0}+\alpha_{1} \alpha_{2} n_{0}^{2}+\frac{m^{4} \alpha_{1} \alpha_{2}}{4 \pi^{2}}\left[K_{1}\left(m\left|\mathbf{R}_{1}-\mathbf{R}_{2}\right|\right)\right]^{2} .(3 .
\end{aligned}
$$

and $\theta_{R_{1} R_{2}}$ the polar angle of the vector $\mathbf{R}_{1}-\mathbf{R}_{2}$.

In order to find $G_{--}$, we note that if we interchange $\mathbf{R}_{1}$ and $\mathbf{R}_{2}$ and their adhesivities in the problem for $G_{++}$, we would have a negative impurity at $\mathbf{r}_{1}=\mathbf{R}_{1}$, and a positive one at $\mathbf{r}_{1}=\mathbf{R}_{2}$. Due to this symmetry argument, the function $G_{--}$for one positive and one negative impurity at $\mathbf{r}_{1}=\mathbf{R}_{1}$ and $\mathbf{r}_{1}=$ $\mathbf{R}_{2}$, is the function $G_{++}$with $\mathbf{R}_{1}$ and $\mathbf{R}_{2}$ and their adhesivities interchanged. Then,

$$
G_{--}\left(\mathbf{r}_{1}, \mathbf{r}_{2} ; \mathbf{R}_{1}, \alpha_{1}, \mathbf{R}_{2}, \alpha_{2}\right)=G_{++}\left(\mathbf{r}_{1}, \mathbf{r}_{2} ; \mathbf{R}_{2}, \alpha_{2}, \mathbf{R}_{1}, \alpha_{1}\right) .
$$

with $G_{++}$given in Eq. (3.25).

Figure 9 shows the charge density profile for this situation.

\subsubsection{General structure of the Green functions for two impurities}

The results of the two previous sections can be put in more general and compact form. Let $S_{1}= \pm 1$ be the sign of the particles the impurity at $\mathbf{R}_{1}$ attracts and $S_{2}= \pm 1$ the sign of the particles the second impurity at $\mathbf{R}_{2}$ attracts. Let us denote by $\hat{G}_{s_{1} s_{2}}=G_{s_{1} s_{2}}^{0}+G_{s_{1} s_{2}}^{1}$, the Green functions for the case of only one impurity located at $\mathbf{R}_{1}$ (Eqs. (3.10) and (3.11)). Notice that in general Eqs. (3.10) and (3.11) can be written as

$$
\hat{G}_{s_{1} s_{2}}\left(\mathbf{r}_{1}, \mathbf{r}_{2}\right)=G_{s_{1} s_{2}}^{0}\left(\mathbf{r}_{1}, \mathbf{r}_{2}\right)-\frac{m \alpha_{1}}{1+\alpha_{1} n_{S_{1}}^{0}\left(\mathbf{R}_{1}\right)} G_{s_{1} S_{1}}^{0}\left(\mathbf{r}_{1}, \mathbf{R}_{1}\right) G_{S_{1} s_{2}}^{0}\left(\mathbf{R}_{1}, \mathbf{r}_{2}\right)
$$

in terms of the Green functions $G_{s_{1} s_{2}}^{0}$ and the density $n_{s}^{0}$ of the unperturbed system.

The solutions for the Green functions found in the two previous sections for the case of two impurities can be written, in term of the Green function for one impurity, as

$$
G_{s_{1} s_{2}}\left(\mathbf{r}_{1}, \mathbf{r}_{2}\right)=\hat{G}_{s_{1} s_{2}}\left(\mathbf{r}_{1}, \mathbf{r}_{2}\right)-\frac{m \alpha_{2}}{\eta} \hat{G}_{s_{1} S_{2}}\left(\mathbf{r}_{1}, \mathbf{R}_{2}\right) \hat{G}_{S_{2} s_{2}}\left(\mathbf{R}_{2}, \mathbf{r}_{2}\right)
$$

where $\eta$ was defined in Eqs. (3.21) and (3.28) (denoted $\bar{\eta}$ in this last equation). In general

$$
\eta=1+\alpha_{1} n_{S_{1}}^{0}\left(\mathbf{R}_{1}\right)+\alpha_{2} n_{S_{2}}^{0}\left(\mathbf{R}_{2}\right)+\alpha_{1} \alpha_{2} n_{S_{1} S_{2}}^{0,(2)}\left(\mathbf{R}_{1}, \mathbf{R}_{2}\right)
$$




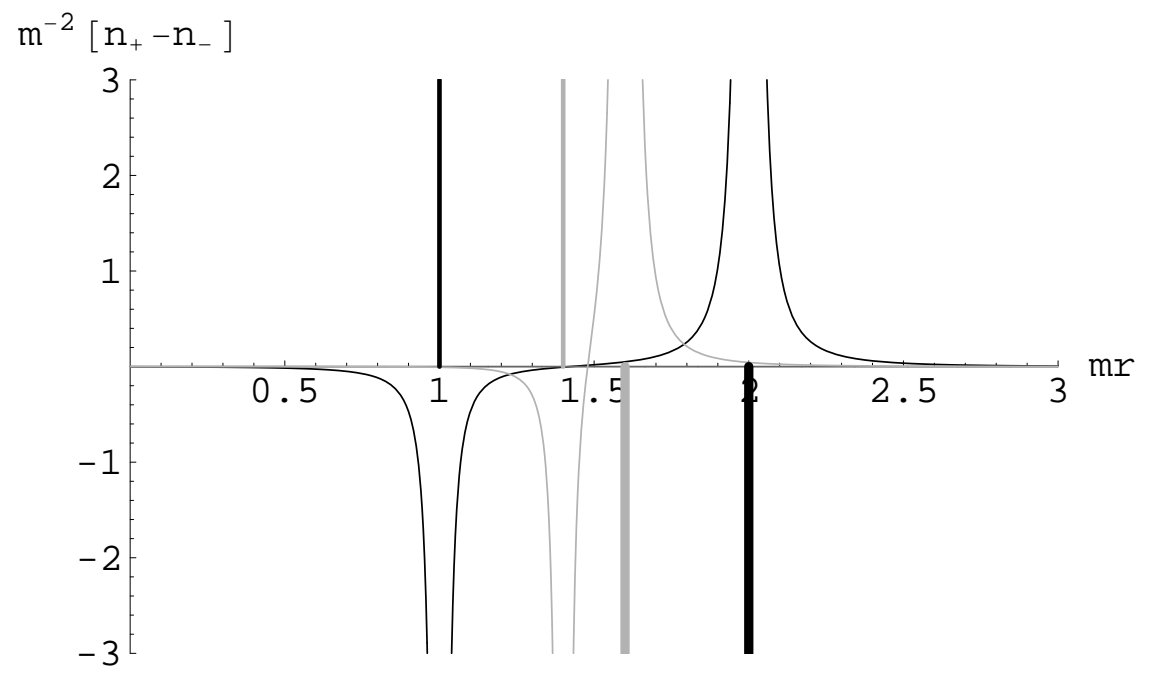

Figure 9: Charge density profile for one "positive" impurity and one "negative" impurity. The adhesivities are $m^{2} \alpha_{1}=0.2$ (positive impurity) and $m^{2} \alpha_{2}=0.5$ (negative impurity). The positions of the impurities are on the $x$ axis at $m R_{1}=$ $1, m R_{1}=1.4$ (positive impurity) and $m R_{2}=2, m R_{2}=1.6$ (negative impurity), respectively from the darkest to the lightest. All the angular differences are zero. The cutoff is $m a=0.01$. 
where $n_{S_{1} S_{2}}^{0,(2)}\left(\mathbf{R}_{1}, \mathbf{R}_{2}\right)$ is the (non-truncated) pair correlation function for the unperturbed system. Notice the similarity in the structure of the Green functions in Eqs. (3.30) and (3.31).

Replacing Eq. (3.30) into Eq. (3.31) gives the expression

$$
\begin{aligned}
G_{s_{1} s_{2}}\left(\mathbf{r}_{1}, \mathbf{r}_{2}\right)= & G_{s_{1} s_{2}}^{0}\left(\mathbf{r}_{1}, \mathbf{r}_{2}\right) \\
- & \frac{1}{\eta}\left[m \alpha_{1}\left[1+\alpha_{2} n_{S_{2}}^{0}\left(\mathbf{R}_{2}\right)\right] G_{s_{1} S_{1}}^{0}\left(\mathbf{r}_{1}, \mathbf{R}_{1}\right) G_{S_{1} s_{2}}^{0}\left(\mathbf{R}_{1}, \mathbf{r}_{2}\right)\right. \\
& +m \alpha_{2}\left[1+\alpha_{1} n_{S_{1}}^{0}\left(\mathbf{R}_{1}\right)\right] G_{s_{1} S_{2}}^{0}\left(\mathbf{r}_{1}, \mathbf{R}_{2}\right) G_{S_{2} s_{2}}^{0}\left(\mathbf{R}_{2}, \mathbf{r}_{2}\right) \\
& -m^{2} \alpha_{1} \alpha_{2}\left[G_{s_{1} S_{1}}^{0}\left(\mathbf{r}_{1}, \mathbf{R}_{1}\right) G_{S_{1} S_{2}}^{0}\left(\mathbf{R}_{1}, \mathbf{R}_{2}\right) G_{S_{2} s_{2}}^{0}\left(\mathbf{R}_{2}, \mathbf{r}_{2}\right)\right. \\
& \left.\left.\quad+G_{s_{1} S_{2}}^{0}\left(\mathbf{r}_{1}, \mathbf{R}_{2}\right) G_{S_{2} S_{1}}^{0}\left(\mathbf{R}_{2}, \mathbf{R}_{1}\right) G_{S_{1} s_{2}}^{0}\left(\mathbf{R}_{1}, \mathbf{r}_{2}\right)\right]\right]
\end{aligned}
$$

in which the exchange symmetry between $\left(\mathbf{R}_{1}, S_{1}\right)$ and $\left(\mathbf{R}_{2}, S_{2}\right)$ is manifest.

\subsection{General results}

The grand potential of the system with impurities can be found by solving the eigenvalue problem (2.6) for each particular situation. We will proceed this way to compute the grand potential for the Coulomb gas in the presence of a continuous adsorbing line in subsections 4.2.1 and 4.2.2. Although this method can also be used to compute the grand potential with point impurities [13], for this latter case, we shall proceed differently, using the general theory developed in Ref. [8] for a one-component fluid with adsorbing sites.

In the following subsections, we obtain expressions for the grand potential and the one- and two-body densities of the plasma with impurities in terms of those same quantities for the plasma without impurities (the unperturbed system). These results are very general: they are valid for any value of the coupling $\Gamma$, they are even valid for any other type of interaction potential between the particles of the system, not only for the Coulomb system considered here. In the present case, since at $\Gamma=2$ the grand potential and density functions for the unperturbed system are known, one can obtain explicit expressions for the thermodynamics and correlations of the plasma with impurities.

\subsubsection{Grand partition function with an arbitrary number of impuri- ties}

Suppose there are $M_{+}$impurities attracting positive particles, located at $\mathbf{R}_{1}^{+}, \ldots, \mathbf{R}_{M_{+}}^{+}$, with adhesivities $\alpha_{1}^{+}, \ldots, \alpha_{M_{+}}^{+}$, and $M_{-}$impurities attracting negative particles, located at $\mathbf{R}_{1}^{-}, \ldots, \mathbf{R}_{M_{-}}^{-}$, with adhesivities $\alpha_{1}^{-}, \ldots, \alpha_{M_{-}}^{-}$. The general calculations done in Ref. [8] for a one-component plasma, can directly be adapted to the present two-component case, to find that the grand partition function $\Xi$ can 
be written as

$$
\begin{gathered}
\Xi=\Xi_{0} \sum_{s_{+}=0}^{\infty} \sum_{s_{-}=0}^{\infty} \sum_{k_{1}^{+}, \ldots, k_{s_{+}}^{+}=0}^{M_{+}} \sum_{k_{1}^{-}, \ldots, k_{s_{-}}^{-}=0}^{M_{-}} \frac{\prod_{n=1}^{s_{+}} \alpha_{k_{n}^{+}} \prod_{n=1}^{s_{-}} \alpha_{k_{n}^{-}}}{s_{+} ! s_{-} !} \\
n_{\left\{s_{+}\right\}\left\{s_{-}\right\}}^{0,\left(s_{+}+s_{-}\right)}\left(\mathbf{R}_{k_{1}^{+}}^{+}, \ldots, \mathbf{R}_{k_{s_{+}}^{+}}^{+}, \mathbf{R}_{k_{1}^{-}}^{-}, \ldots, \mathbf{R}_{k_{s_{-}}^{-}}^{-}\right)
\end{gathered}
$$

where $\Xi_{0}$ is the grand partition function of the unperturbed system and $n_{\left\{s_{+}\right\}\left\{s_{-}\right\}}^{0,\left(s_{+}+s_{-}\right)}$ the $\left(s_{+}+s_{-}\right)$-body density for $s_{+}$positive particles and $s_{-}$negative particles of the unperturbed system. Notice that, since the correlation function vanishes if two of its arguments are equal, the above expression is not an infinite sum, it contains at most terms involving the $\left(M_{+}+M_{-}\right)$-body correlation function and lower degree correlations.

From expression (3.34) we can obtain the density and $k$-body correlation functions performing successive functional derivations of the grand partition function with respect to the fugacity.

\subsubsection{One impurity}

In the case of a single impurity located at $\mathbf{R}_{1}$, with adhesivity $\alpha_{1}$ and attracting particles of sign $S_{1}$, Eq. (3.34) simply reduces to

$$
\Xi=\left[1+\alpha_{1} n_{S_{1}}\left(\mathbf{R}_{1}\right)\right] \Xi_{0} .
$$

Then, the grand potential $\Omega=-k_{B} T \ln \Xi$ can be written as $\Omega=\Omega_{0}+\Omega_{\mathrm{exc}}\left(\alpha_{1}\right)$ with $\Omega_{0}$ the grand potential of the unperturbed system and an excess grand potential

$$
\beta \Omega_{\mathrm{exc}}\left(\alpha_{1}\right)=-\ln \left[1+\alpha_{1} n_{S_{1}}\left(\mathbf{R}_{1}\right)\right] .
$$

As explained in Ref. [8], the adhesivity $\alpha$ can be interpreted also as a fugacity for the adsorbed particles. Thus, one can compute the average number of adsorbed particles from the relation

$$
N_{S_{1}}^{\alpha_{1}}=-\alpha_{1} \frac{\partial \beta \Omega_{\mathrm{exc}}\left(\alpha_{1}\right)}{\partial \alpha_{1}}=\frac{\alpha_{1} n_{S_{1}}^{0}\left(\mathbf{R}_{1}\right)}{1+\alpha_{1} n_{S_{1}}^{0}\left(\mathbf{R}_{1}\right)} .
$$

We recover the result (3.14) obtained from a direct calculation at $\Gamma=2$.

From Eq. (3.35) we can also rederive the results from the previous section for the density and correlation functions, by performing functional derivatives of $\Xi$ with respect to the fugacity. Remembering that

$$
\left.m_{s}(\mathbf{r}) \frac{\delta \ln \Xi_{0}}{\delta m_{s}(\mathbf{r})}\right|_{m(\mathbf{r})=m}=n_{s}^{0}(\mathbf{r})
$$

and

$$
\left.m_{s_{2}}\left(\mathbf{r}_{2}\right) \frac{\delta n_{s_{1}}^{0}\left(\mathbf{r}_{1}\right)}{\delta m_{s_{2}}\left(\mathbf{r}_{2}\right)}\right|_{m(\mathbf{r})=m}=n_{s_{1} s_{2}}^{0,(2) T}\left(\mathbf{r}_{1}, \mathbf{r}_{2}\right)
$$


deriving (3.35) with respect to $m_{s}(\mathbf{r})$, we obtain

$$
n_{S_{1}}(\mathbf{r})=\left[1+\alpha_{1} \delta\left(\mathbf{r}-\mathbf{R}_{1}\right)\right]\left[n_{S_{1}}^{0}(\mathbf{r})+\frac{\alpha_{1} n_{S_{1} S_{1}}^{0,(2) T}\left(\mathbf{r}, \mathbf{R}_{1}\right)}{1+\alpha_{1} n_{S_{1}}^{0}\left(\mathbf{R}_{1}\right)}\right]
$$

and

$$
n_{-S_{1}}(\mathbf{r})=n_{-S_{1}}^{0}(\mathbf{r})+\frac{\alpha_{1} n_{-S_{1} S_{1}}^{0,(2) T}\left(\mathbf{r}, \mathbf{R}_{1}\right)}{1+\alpha_{1} n_{S_{1}}^{0}\left(\mathbf{R}_{1}\right)} .
$$

When $\Gamma=2$, we recover the results (3.12) from section 3.1.1. Deriving Eqs. (3.40) once again with respect to the fugacity, we obtain the truncated two-body correlation functions

$$
\begin{aligned}
n_{S_{1} S_{1}}^{(2) T}\left(\mathbf{r}_{1}, \mathbf{r}_{2}\right) & =\left[1+\alpha_{1} \delta\left(\mathbf{r}_{1}-\mathbf{R}_{1}\right)\right]\left[1+\alpha_{1} \delta\left(\mathbf{r}_{2}-\mathbf{R}_{1}\right)\right] n_{S_{1} S_{1}}^{(2) T *}\left(\mathbf{r}_{1}, \mathbf{r}_{2}\right) \\
n_{-S_{1} S_{1}}^{(2) T}\left(\mathbf{r}_{1}, \mathbf{r}_{2}\right) & =\left[1+\alpha_{1} \delta\left(\mathbf{r}_{2}-\mathbf{R}_{1}\right)\right] n_{-S_{1} S_{1}}^{(2) T *}\left(\mathbf{r}_{1}, \mathbf{r}_{2}\right) \\
n_{-S_{1},-S_{1}}^{(2) T}\left(\mathbf{r}_{1}, \mathbf{r}_{2}\right) & =n_{-S_{1},-S_{1}}^{(2) T *}\left(\mathbf{r}_{1}, \mathbf{r}_{2}\right)
\end{aligned}
$$

with

$$
\begin{aligned}
n_{s_{1} s_{2}}^{(2) T *}\left(\mathbf{r}_{1}, \mathbf{r}_{2}\right)= & n_{s_{1} s_{2}}^{0,(2) T}\left(\mathbf{r}_{1}, \mathbf{r}_{2}\right)-\frac{\alpha_{1}^{2} n_{s_{1} S_{1}}^{0,(2) T}\left(\mathbf{r}_{1}, \mathbf{R}_{1}\right) n_{S_{1} s_{2}}^{0,(2) T}\left(\mathbf{R}_{1}, \mathbf{r}_{2}\right)}{\left[1+\alpha_{1} n_{S_{1}}^{0}\left(\mathbf{R}_{1}\right)\right]^{2}} \\
& +\frac{\alpha_{1} n_{s_{1} S_{2} S_{1}}^{0,(3) T}\left(\mathbf{r}_{1}, \mathbf{r}_{2}, \mathbf{R}_{1}\right)}{1+\alpha_{1} n_{S_{1}}^{0}\left(\mathbf{R}_{1}\right)}
\end{aligned}
$$

where $n_{s_{1} s_{2} s_{3}}^{0,(3) T}$ is the truncated three-body density function of the unperturbed system. From the Green functions, Eq. (3.30), obtained in the previous section and the general relations (2.3), it can be shown that the correlation functions obtained in section 3.1.1 are indeed those given by Eqs. (3.41) and (3.42) when $\Gamma=2$.

\subsubsection{Two impurities}

Now, let us consider the case of two impurities, located at $\mathbf{R}_{1}$ and $\mathbf{R}_{2}$ with adhesivities $\alpha_{1}$ and $\alpha_{2}$ and attracting particles of sign $S_{1}$ and $S_{2}$, respectively. Eq. (3.34) gives

$$
\Xi=\Xi_{0}\left[1+\alpha_{1} n_{S_{1}}^{0}\left(\mathbf{R}_{1}\right)+\alpha_{2} n_{S_{2}}^{0}\left(\mathbf{R}_{2}\right)+\alpha_{1} \alpha_{2} n_{S_{1} S_{2}}^{0,(2)}\left(\mathbf{R}_{1}, \mathbf{R}_{2}\right)\right]=\Xi_{0} \eta
$$

with $\eta$ defined in Eq. (3.32). The grand potential now reads

$$
\beta \Omega=\beta \Omega_{0}-\ln \eta .
$$

Notice that in the problems for one and two impurities the excess grand potential is the logarithm of the denominator of the Green functions $G_{s_{1} s_{2}}^{1}$ and $G_{s_{1} s_{2}}^{2}$ respectively. It is convenient to express the grand potential as

$$
\Omega=\Omega_{0}+\Omega_{\mathrm{exc}}\left(\alpha_{1}\right)+\Omega_{\mathrm{exc}}\left(\alpha_{2}\right)+\Omega_{S_{1} S_{2}}\left(\mathbf{R}_{1}, \alpha_{1} ; \mathbf{R}_{2}, \alpha_{2}\right)
$$




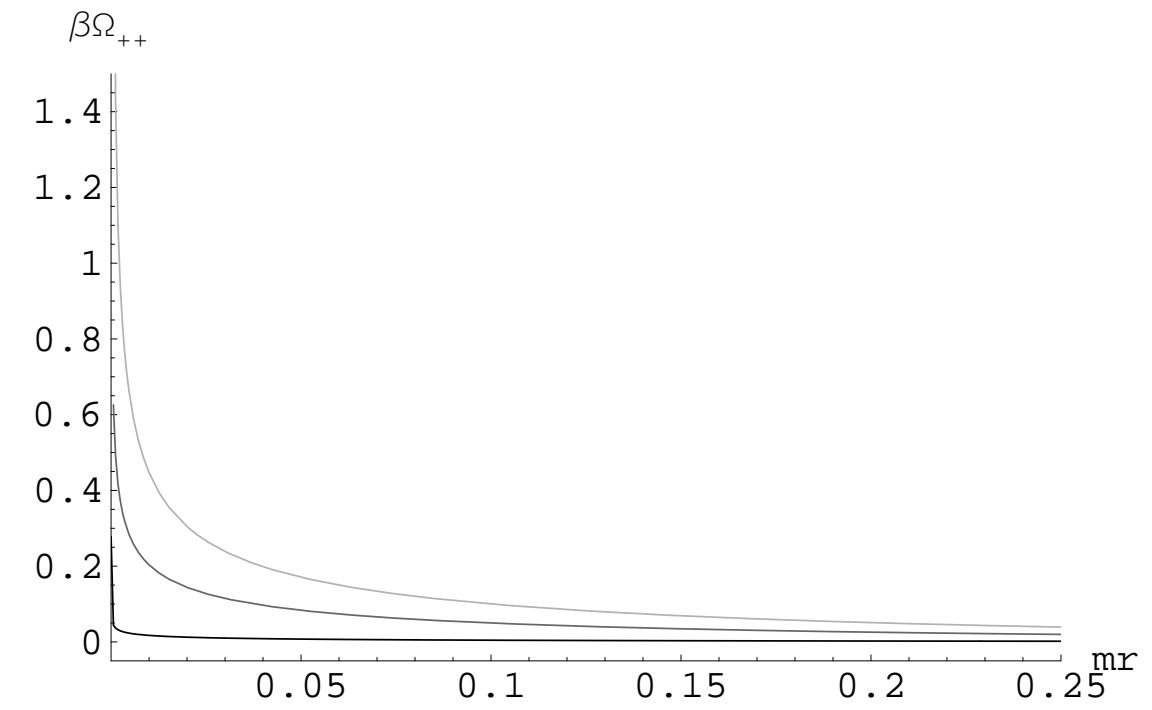

Figure 10: Effective interaction between two positive impurities as a function of their distance. The adhesivities are $m^{2} \alpha_{1}=m^{2} \alpha_{2}=0.2,1$ and 2 from darkest to lightest. The cutoff is $m a=0.01$.

with

$$
\beta \Omega_{S_{1} S_{2}}\left(\mathbf{R}_{1}, \alpha_{1} ; \mathbf{R}_{2}, \alpha_{2}\right)=-\ln \left[1+\frac{\alpha_{1} \alpha_{2} n_{S_{1} S_{2}}^{0,(2) T}\left(\mathbf{R}_{1}, \mathbf{R}_{2}\right)}{\left[1+\alpha_{1} n_{S_{1}}^{0}\left(\mathbf{R}_{1}\right)\right]\left[1+\alpha_{2} n_{S_{2}}^{0}\left(\mathbf{R}_{2}\right)\right]}\right]
$$

Explicitly, for $\Gamma=2$,

$$
\beta \Omega_{ \pm \pm}\left(\mathbf{R}_{1}, \alpha_{1} ; \mathbf{R}_{2}, \alpha_{2}\right)=-\ln \left[1-\frac{m^{4} \alpha_{1} \alpha_{2}\left[K_{0}\left(m\left|\mathbf{R}_{1}-\mathbf{R}_{2}\right|\right)\right]^{2}}{4 \pi^{2}\left[1+\alpha_{1} n_{0}\right]\left[1+\alpha_{2} n_{0}\right]}\right]
$$

and

$$
\beta \Omega_{ \pm \mp}\left(\mathbf{R}_{1}, \alpha_{1} ; \mathbf{R}_{2}, \alpha_{2}\right)=-\ln \left[1+\frac{m^{4} \alpha_{1} \alpha_{2}\left[K_{1}\left(m\left|\mathbf{R}_{1}-\mathbf{R}_{2}\right|\right)\right]^{2}}{4 \pi^{2}\left[1+\alpha_{1} n_{0}\right]\left[1+\alpha_{2} n_{0}\right]}\right] .
$$

A work $\Omega_{\text {exc }}\left(\alpha_{i}\right)$ is required to introduce a single impurity into the plasma. To introduce two impurities, we require, in addition to $\Omega_{\text {exc }}\left(\alpha_{1}\right)+\Omega_{\text {exc }}\left(\alpha_{2}\right)$, an additional work $\Omega_{S_{1} S_{2}}\left(\mathbf{R}_{1}, \alpha_{1} ; \mathbf{R}_{2}, \alpha_{2}\right)$. We can define the effective chemical potential of an impurity with adhesivity $\alpha$ as $\mu(\alpha)=\Omega_{\text {exc }}(\alpha)$. The term $\Omega_{S_{1} S_{2}}\left(\mathbf{R}_{1}, \alpha_{1} ; \mathbf{R}_{2}, \alpha_{2}\right)$ represents an effective interaction between the impurities. This contribution is not a direct interaction between the adsorbing particles but a consequence of the inhomogeneous charge distribution that they create. 


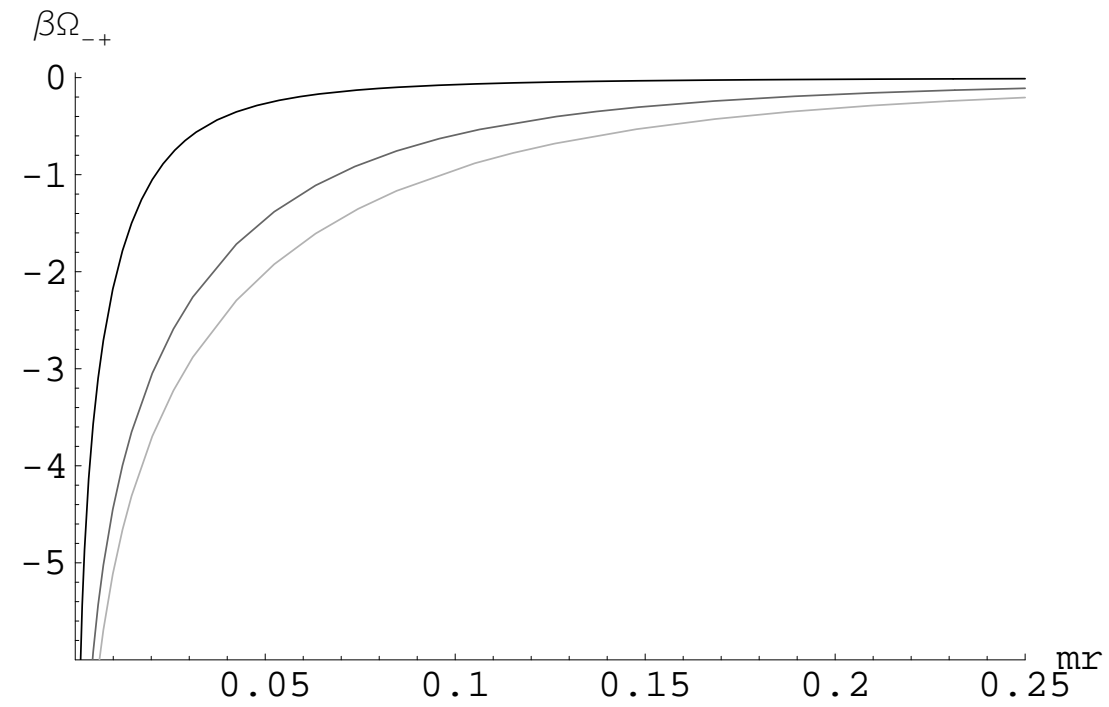

Figure 11: Effective interaction between a positive and a negative impurity as a function of their distance. The values are the same than in figure 10 .

Figures 10 and 11 show the effective interaction between two adsorbing particles. It can be seen that it is repulsive if the impurities have the same sign and attractive if their sign is opposite. As expected, the interaction is important when the impurities are close enough but is negligible for large distances. It depends on the adhesivity of the two impurities, indeed, it is larger for large adhesivities.

From Eq. (3.43), we can obtain the density profiles by performing a functional derivative with respect to the fugacity. We obtain

$$
n_{s}(\mathbf{r})= \begin{cases}{\left[1+\alpha_{1} \delta\left(\mathbf{r}-\mathbf{R}_{1}\right)+\alpha_{2} \delta\left(\mathbf{r}-\mathbf{R}_{2}\right)\right] n_{s}^{*}(\mathbf{r})} & \text { if } s=S_{1}=S_{2} \\ {\left[1+\alpha_{1} \delta\left(\mathbf{r}-\mathbf{R}_{1}\right)\right] n_{s}^{*}(\mathbf{r})} & \text { if } s=S_{1}=-S_{2} \\ {\left[1+\alpha_{2} \delta\left(\mathbf{r}-\mathbf{R}_{2}\right)\right] n_{s}^{*}(\mathbf{r})} & \text { if } s=S_{2}=-S_{1} \\ n_{s}^{*}(\mathbf{r}) & \text { if } s=-S_{1}=-S_{2}\end{cases}
$$

with

$$
\begin{aligned}
n_{s}^{*}(\mathbf{r}) & =n_{s}^{0}(\mathbf{r})+\frac{1}{\eta}\left[\alpha_{1} n_{s S_{1}}^{0,(2) T}\left(\mathbf{r}, \mathbf{R}_{1}\right)\left[1+\alpha_{2} n_{S_{2}}^{0}\left(\mathbf{R}_{2}\right)\right]\right. \\
& \left.+\alpha_{2} n_{s S_{2}}^{0,(2) T}\left(\mathbf{r}, \mathbf{R}_{2}\right)\left[1+\alpha_{1} n_{S_{1}}^{0}\left(\mathbf{R}_{1}\right)\right]+\alpha_{1} \alpha_{2} n_{s S_{1} S_{2}}^{0,(3) T}\left(\mathbf{r}, \mathbf{R}_{1}, \mathbf{R}_{2}\right)\right]
\end{aligned}
$$

With the Green functions (3.33) for two impurities found in sections 3.1.2, 3.1.3 
and 3.1.4 and the general relation (2.3), it is direct to verify that the density is indeed given by Eqs. (3.49) and (3.50) when $\Gamma=2$.

We can compute the average number of adsorbed particles by each impurity $N_{S_{i}}^{\alpha_{i}}$, for $i=1,2$. This can be obtained either from the relation $N_{S_{i}}^{\alpha_{i}}=$ $\alpha_{i} n_{S_{i}}^{*}\left(\mathbf{R}_{i}\right)$, or from

$$
N_{S_{i}}^{\alpha_{i}}=-\alpha_{i} \frac{\partial(\beta \Omega)}{\partial \alpha_{i}}
$$

We obtain

$$
N_{S_{i}}^{\alpha_{i}}=\frac{\alpha_{i} n_{S_{i}}^{0}\left(\mathbf{R}_{i}\right)+\alpha_{1} \alpha_{2} n_{S_{1} S_{2}}^{0,(2)}\left(\mathbf{R}_{1}, \mathbf{R}_{2}\right)}{1+\alpha_{1} n_{S_{1}}^{0}\left(\mathbf{R}_{1}\right)+\alpha_{2} n_{S_{2}}^{0}\left(\mathbf{R}_{2}\right)+\alpha_{1} \alpha_{2} n_{S_{1} S_{2}}^{0,(2)}\left(\mathbf{R}_{1}, \mathbf{R}_{2}\right)} .
$$

\section{The plasma with adsorbing lines}

\subsection{Density and correlations}

\subsubsection{An infinite line}

As a simple model for electrodes with adsorbing sites, we consider now that a line of impurities is introduced into the plasma. We shall consider the case where the impurities are very close to each other, so there is a continuous adsorbing line in the plasma, located at $x=x_{0}$. Since the system is translational invariant in the direction of the line (the $y$ direction), it is better to work in Cartesian coordinates and confine the plasma in a rectangular box. The fugacity can be modeled by

$$
m_{ \pm}(\mathbf{r})=m_{ \pm}(x)=m\left[1+\bar{\alpha}_{ \pm} \delta\left(x-x_{0}\right)\right] .
$$

The adhesivity $\bar{\alpha}_{ \pm}$has length dimensions, unlike the adhesivity $\alpha_{ \pm}$for point impurities which has area dimensions. Actually, $\bar{\alpha}_{ \pm}$is an effective adhesivity due to the distribution of the adsorbing particles on the line. We will assume that the line is composed of "positive" impurities, so $\bar{\alpha}_{+}=\bar{\alpha}_{1}$ and $\bar{\alpha}_{-}=0$.

Due to the translational invariance in the $y$ direction, this problem is easier to solve via Fourier transform. For this purpose we define

$$
G_{s_{1} s_{2}}\left(\mathbf{r}_{1}, \mathbf{r}_{2}\right)=\frac{1}{2 \pi} \int_{\mathbb{R}} \widetilde{G}_{s_{1} s_{2}}\left(x_{1}, x_{2}, l\right) e^{i l\left(y_{1}-y_{2}\right)} d l .
$$

By replacing Eq. (4.2) and the given fugacities into Eqs. (2.2), we obtain the 
system

$$
\begin{aligned}
m\left[1+\bar{\alpha}_{1} \delta\left(x_{1}-x_{0}\right)\right] \widetilde{G}_{++}\left(x_{1}, x_{2}, l\right) & \\
+\left(\frac{d}{d x_{1}}+l\right) \widetilde{G}_{-+}\left(x_{1}, x_{2}, l\right) & =\delta\left(x_{1}-x_{2}\right) \\
\left(\frac{d}{d x_{1}}-l\right) \widetilde{G}_{++}\left(x_{1}, x_{2}, l\right)+m \widetilde{G}_{-+}\left(x_{1}, x_{2}, l\right) & =0 \\
m\left[1+\bar{\alpha}_{1} \delta\left(x_{1}-x_{0}\right)\right] \widetilde{G}_{+-}\left(x_{1}, x_{2}, l\right) & \\
+\left(\frac{d}{d x_{1}}+l\right) \widetilde{G}_{--}\left(x_{1}, x_{2}, l\right) & =0 \\
\left(\frac{d}{d x_{1}}-l\right) \widetilde{G}_{+-}\left(x_{1}, x_{2}, l\right)+m \widetilde{G}_{--}\left(x_{1}, x_{2}, l\right) & =\delta\left(x_{1}-x_{2}\right) .
\end{aligned}
$$

We assume solutions of the form $\widetilde{G}_{s_{1} s_{2}}=\widetilde{G}_{s_{1} s_{2}}^{0}+\widetilde{G}_{s_{1} s_{2}}^{1}$ where $\widetilde{G}_{s_{1} s_{2}}^{0}$ are the bulk solutions for $\bar{\alpha}_{1}=0$. It is easy to show that these solutions are given by [1]

$$
\begin{aligned}
& \widetilde{G}_{ \pm \pm}^{0}\left(x_{1}, x_{2}, l\right)=\frac{m}{2 k} e^{-k\left|x_{1}-x_{2}\right|} \\
& \widetilde{G}_{-+}^{0}\left(x_{1}, x_{2}, l\right)=\frac{1}{m}\left(-\frac{d}{d x_{1}}+l\right) \widetilde{G}_{++}^{0}\left(x_{1}, x_{2}, l\right) \\
& \widetilde{G}_{+-}^{0}\left(x_{1}, x_{2}, l\right)=-\frac{1}{m}\left(\frac{d}{d x_{1}}+l\right) \widetilde{G}_{--}^{0}\left(x_{1}, x_{2}, l\right)
\end{aligned}
$$

with $k=\sqrt{m^{2}+l^{2}}$.

The solutions to Eqs. (4.3) are

$$
\begin{aligned}
& \widetilde{G}_{++}\left(x_{1}, x_{2}, l\right)=\left\{\begin{array}{cc}
\frac{m}{2 k} e^{-k\left|x_{1}-x_{2}\right|}-\frac{m^{3} \bar{\alpha}_{1}}{2 k\left(2 k+m^{2} \bar{\alpha}_{1}\right)} e^{-k\left|2 x_{0}-x_{1}-x_{2}\right|}, & x_{0}<x_{1}, x_{2}, \\
\frac{m}{2 k+m^{2} \bar{\alpha}_{1}} e^{-k\left|x_{1}-x_{2}\right|} & \text { or } x_{0}>x_{1}, x_{2} \\
x_{0} \text { between } x_{1} \text { and } x_{2}
\end{array}\right. \\
& \widetilde{G}_{--}\left(x_{1}, x_{2}, l\right)=\left\{\begin{array}{cc}
\frac{m}{2 k} e^{-k\left|x_{1}-x_{2}\right|}+\frac{m \bar{\alpha}_{1}(k+l)^{2}}{2 k\left(2 k+m^{2} \bar{\alpha}_{1}\right)} e^{k\left(2 x_{0}-x_{1}-x_{2}\right)}, & x_{0}<x_{1}, x_{2} \\
\frac{m}{2 k+m^{2} \bar{\alpha}_{1}} e^{-k\left|x_{1}-x_{2}\right|}, & x_{0} \text { between } x_{1} \text { and } x_{2} \\
\frac{m}{2 k} e^{-k\left|x_{1}-x_{2}\right|}+\frac{m \bar{\alpha}_{1}(k-l)^{2}}{2 k\left(2 k+m^{2} \bar{\alpha}_{1}\right)} e^{-k\left(2 x_{0}-x_{1}-x_{2}\right)}, & x_{0}>x_{1}, x_{2}
\end{array}\right.
\end{aligned}
$$

The number density profiles $n_{+}, n_{-}$, and charge density profile $\rho$ (in units of $e$ ) are obtained using Eqs. (2.3). We find

$$
\begin{aligned}
n_{+}(x) & =\left[1+\bar{\alpha}_{1} \delta\left(x-x_{0}\right)\right] n_{+}^{*}(x) \\
n_{-}(x) & =n_{-}^{*}(x) \\
\rho(x) & =n_{+}(x)-n_{-}(x)=\bar{\alpha}_{1} \delta\left(x-x_{0}\right) n_{+}^{*}\left(x_{0}\right)+\rho^{*}(x)
\end{aligned}
$$




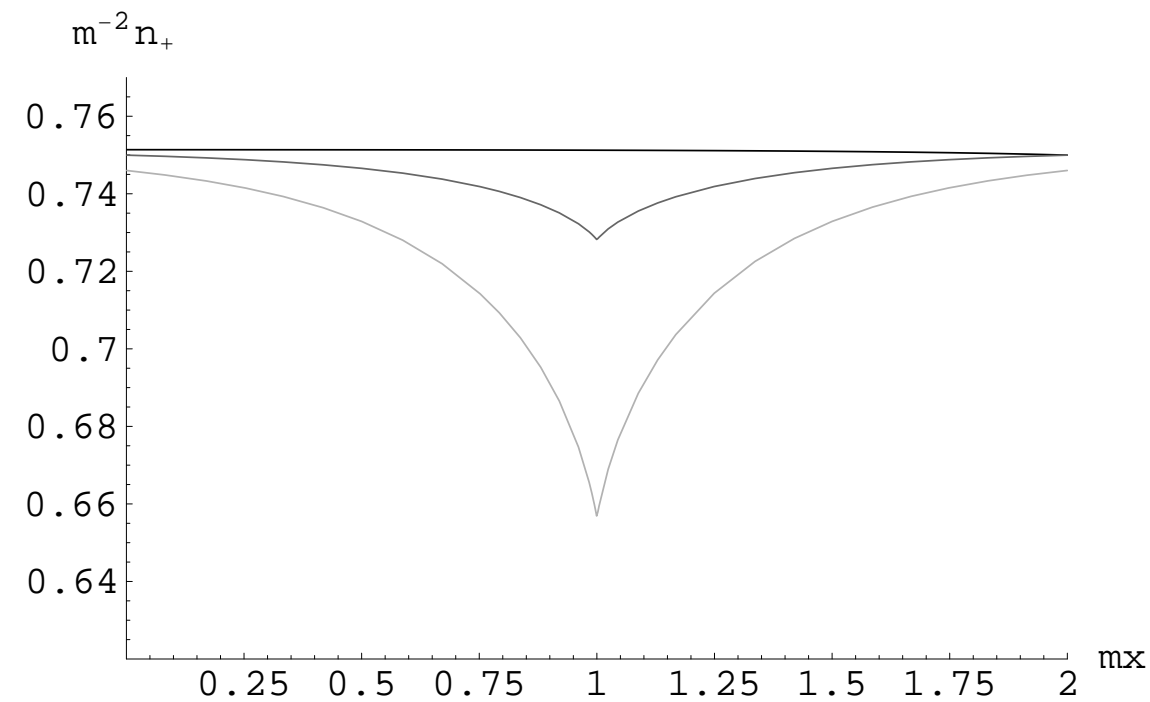

Figure 12: Positive density for a line of "positive" impurities at $m x=1$. The adhesivities are $m \bar{\alpha}_{1}=0.2,1$ and 3 from the darkest to the lightest. The Dirac distribution at $m x=1$ is not plotted. The cutoff is $m a=0.01$.

with

$$
\begin{aligned}
n_{+}^{*}(x) & =n_{+}^{0}-m^{3} \bar{\alpha}_{1} \int_{0}^{\infty} \frac{d t}{4 \pi} \frac{e^{-2 \sqrt{t^{2}+1} m\left|x-x_{0}\right|}}{\sqrt{t^{2}+1}\left(\sqrt{t^{2}+1}+\tilde{\alpha}\right)} \\
n_{-}^{*}(x) & =n_{-}^{0}+m^{3} \bar{\alpha}_{1} \int_{0}^{\infty} \frac{d t}{4 \pi} \frac{\left(1+2 t^{2}\right) e^{-2 \sqrt{t^{2}+1}} m\left|x-x_{0}\right|}{\sqrt{t^{2}+1}\left(\sqrt{t^{2}+1}+\tilde{\alpha}\right)} \\
\rho^{*}(x) & =-m^{3} \bar{\alpha}_{1} \int_{0}^{\infty} \frac{d t}{2 \pi} \frac{\sqrt{t^{2}+1} e^{-2 \sqrt{t^{2}+1} m\left|x-x_{0}\right|}}{\sqrt{t^{2}+1}+\tilde{\alpha}}
\end{aligned}
$$

with $\tilde{\alpha}=m \bar{\alpha}_{1} / 2$.

Figures 12,13 and 14 show the positive and negative density and the charge density profile for this configuration. The curves obtained are similar to the ones obtained for point impurities. They differ in the magnitude as well as in the peaks near the adsorbing particles. The magnitude of the density at the adsorbing line is reduced as a consequence of the impurities distribution.

The linear charge density adsorbed by the line, $\sigma$, can be computed by the relation

$$
\sigma=\bar{\alpha}_{1} n_{+}^{*}\left(x_{0}\right)
$$

or by integrating the opposite of the nonadsorbed charge density

$$
\sigma=-\int_{-\infty}^{\infty} \rho^{*}(x) d x
$$




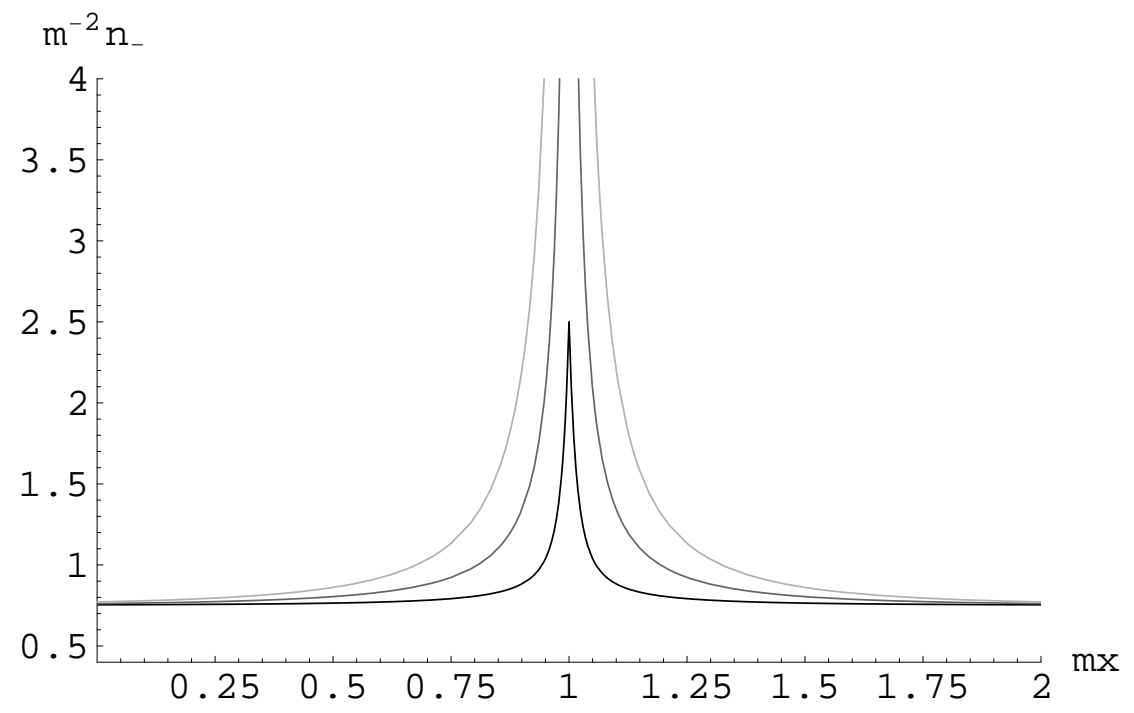

Figure 13: Negative density for a line of "positive" impurities at $m x=1$. The adhesivities are $m \bar{\alpha}_{1}=0.2,1$ and 3 from the darkest to the lightest. The cutoff is $m a=0.01$.

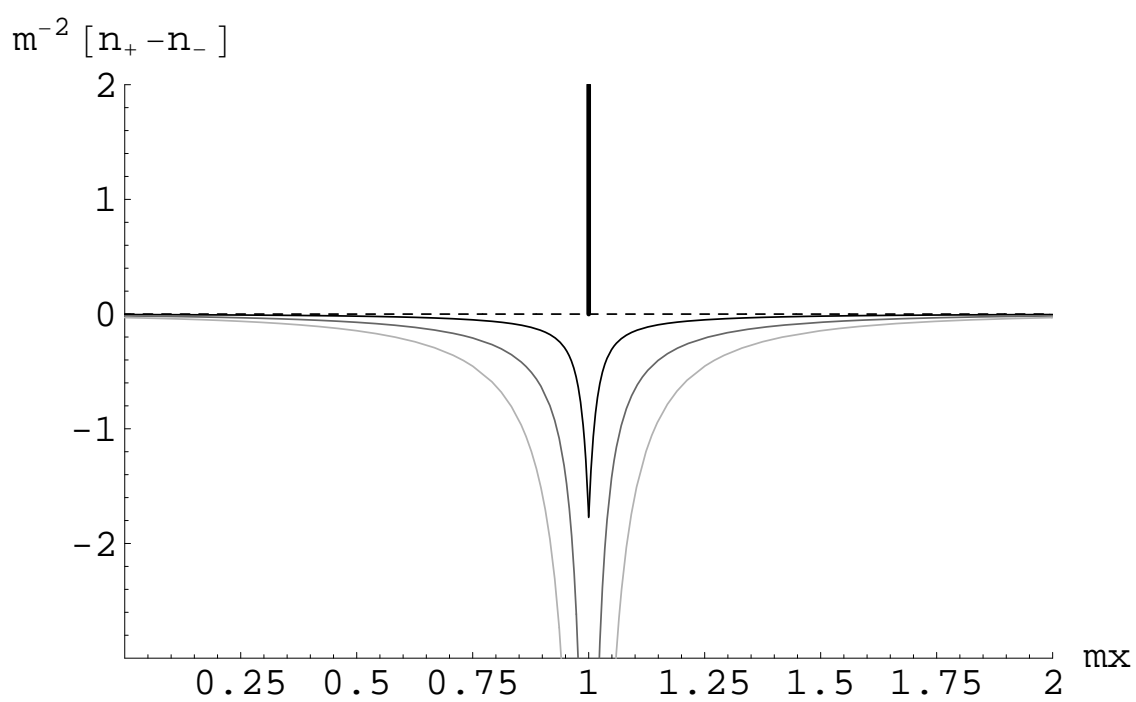

Figure 14: Charge density profile for a line of "positive" impurities at $m x=1$. The adhesivities are $m \bar{\alpha}_{1}=0.2,1$ and 3 from the darkest to the lightest. The cutoff is $m a=0.01$. 
Either way, the result is the same, as expected,

$$
\begin{aligned}
\sigma & =m^{2} \bar{\alpha}_{1} \int_{0}^{t_{\max }} \frac{d t}{2 \pi} \frac{1}{\sqrt{t^{2}+1}+\tilde{\alpha}} \\
& =\bar{\alpha}_{1} n_{+}^{0}-\frac{m}{\pi} \frac{\tilde{\alpha}^{2}}{\sqrt{\tilde{\alpha}^{2}-1}} \operatorname{arctanh} \frac{\sqrt{\tilde{\alpha}^{2}-1}}{\tilde{\alpha}}
\end{aligned}
$$

where we introduced an ultraviolet cutoff $t_{\max }=e^{-C} /(m a)$ to obtain a finite result. This choice of the cutoff is done to ensure the correct result for bulk density [1].

Contrary to the case of a point adsorbing impurity, where the adsorbed charge reached a maximun value of 1 when $\alpha_{1} \rightarrow \infty$, the adsorbed line charge density is not bounded when $\bar{\alpha}_{1} \rightarrow \infty$. Indeed, for large $\bar{\alpha}_{1}$,

$$
\sigma=\bar{\alpha}_{1} n_{+}^{0}-\frac{m \tilde{\alpha}}{\pi}\left[\ln (2 \tilde{\alpha})+\frac{1}{2 \tilde{\alpha}^{2}}\left[\ln (2 \tilde{\alpha})-\frac{1}{2}\right]+O\left(\tilde{\alpha}^{-4}\right)\right]
$$

It is instructing to compute the electric field $E(x)$ and electric potential $\phi(x)$ created by the adsorbed line charge density and its polarization cloud. Integrating Poisson equation we obtain, for $x>0$ and choosing $x_{0}=0$,

$$
E(x)=e m \tilde{\alpha} \int_{0}^{\infty} \frac{e^{-2 \sqrt{t^{2}+1} m x} d t}{\sqrt{t^{2}+1}+\tilde{\alpha}}
$$

and

$$
\phi(x)=e \tilde{\alpha} \int_{0}^{\infty} \frac{e^{-2 \sqrt{t^{2}+1} m x} d t}{t^{2}+1+\tilde{\alpha} \sqrt{t^{2}+1}}
$$

Notice that the electric field at the position of the impurity is related to the adsorbed linear charge density by $E\left(0^{+}\right)=\pi \sigma$, and as usual we have the expected discontinuity $E\left(0^{+}\right)-E\left(0^{-}\right)=2 \pi \sigma$. The potential drop from the adsorbed line to infinity is

$$
\phi(0)-\phi(+\infty)=e \tilde{\alpha} \int_{0}^{\infty} \frac{d t}{t^{2}+1+\tilde{\alpha} \sqrt{t^{2}+1}}=\frac{e \tilde{\alpha}}{\sqrt{\tilde{\alpha}^{2}-1}} \operatorname{arctanh} \frac{\sqrt{\tilde{\alpha}^{2}-1}}{\tilde{\alpha}} .
$$

The correlation functions can be obtained from the Green functions Eq. (4.5) and (4.6), using the relation (2.3b). Let us simply note that the correlation functions have an exponential decay both in the $x$ direction (transverse to the adsorbing line) and the $y$ direction (along the adsorbing line), a behavior similar to the case without adsorbing line. This is different from the algebraic decay that the correlation functions show parallel along a hard wall $[14,15,16]$.

\subsubsection{Two infinite lines}

For two parallel infinite lines it is also possible to find analytic expressions for the Green functions. For two lines located at $x=X_{1}$ and $x=X_{2}$, both attracting 
the positive particles, the fugacities are

$$
\begin{aligned}
& m_{+}(x)=m\left[1+\bar{\alpha}_{1} \delta\left(x-X_{1}\right)+\bar{\alpha}_{2} \delta\left(x-X_{2}\right)\right] \\
& m_{-}(x)=m
\end{aligned}
$$

For one line, located at $x=X_{1}$, attracting the positive particles, and a second line, located at $x=X_{2}$, attracting the negative particles, the fugacities are

$$
\begin{aligned}
& m_{+}(x)=m\left(1+\bar{\alpha}_{1} \delta\left(x-X_{1}\right)\right) \\
& m_{-}(x)=m\left(1+\bar{\alpha}_{2} \delta\left(x-X_{2}\right)\right)
\end{aligned}
$$

Without loss of generality, we suppose that $X_{1}<X_{2}$. The resolution of the linear system (2.2) satisfied by the Green functions follows similar steps as for the previous case of one line. The explicit expressions for the Green functions depend on the relative positions of their arguments with respect to the lines. The complete expressions can be found in Ref. [13]. Let us focus here in the expressions obtained for the density profiles.

For two lines attracting positive particles, we find the positive, negative and charge densities

$$
\begin{aligned}
n_{+}(x) & =\left[1+\bar{\alpha}_{1} \delta\left(x-X_{1}\right)+\bar{\alpha}_{2} \delta\left(x-X_{2}\right)\right] n_{+}^{*}(x) \\
n_{-}(x) & =n_{-}^{*}(x) \\
\rho(x) & =\left[\bar{\alpha}_{1} \delta\left(x-X_{1}\right)+\bar{\alpha}_{2} \delta\left(x-X_{2}\right)\right] n_{+}^{*}(x)+\rho^{*}(x)
\end{aligned}
$$

with

$$
\begin{aligned}
& n_{+}^{*}(x)=n_{+}^{0}-\int_{-\infty}^{\infty} \frac{m^{4} e^{-2 k x}}{4 \pi k} \frac{\bar{\alpha}_{1}\left(2 k-m^{2} \bar{\alpha}_{2}\right) e^{2 k X_{1}}+\bar{\alpha}_{2}\left(2 k+m^{2} \bar{\alpha}_{1}\right) e^{2 k X_{2}}}{\left(2 k+m^{2} \bar{\alpha}_{1}\right)\left(2 k+m^{2} \bar{\alpha}_{2}\right)-m^{4} \bar{\alpha}_{1} \bar{\alpha}_{2} e^{2 k\left(X_{1}-X_{2}\right)}} d l \text { (4.21a) } \\
& n_{-}^{*}(x)=n_{-}^{0}+\int_{-\infty}^{\infty} \frac{m^{2}(k+l)^{2} e^{-2 k x}}{4 \pi k} \frac{\bar{\alpha}_{1}\left(2 k-m^{2} \bar{\alpha}_{2}\right) e^{2 k X_{1}}+\bar{\alpha}_{2}\left(2 k+m^{2} \bar{\alpha}_{1}\right) e^{2 k X_{2}}}{\left(2 k+m^{2} \bar{\alpha}_{1}\right)\left(2 k+m^{2} \bar{\alpha}_{2}\right)-m^{4} \bar{\alpha}_{1} \bar{\alpha}_{2} e^{2 k\left(X_{1}-X_{2}\right)}} d l \\
& \rho^{*}(x)=-\int_{-\infty}^{+\infty} \frac{m^{2} k e^{-2 k x}}{2 \pi} \frac{\bar{\alpha}_{1}\left(2 k-m^{2} \bar{\alpha}_{2}\right) e^{2 k X_{1}}+\bar{\alpha}_{2}\left(2 k+m^{2} \bar{\alpha}_{1}\right) e^{2 k X_{2}}}{\left(2 k+m^{2} \bar{\alpha}_{1}\right)\left(2 k+m^{2} \bar{\alpha}_{2}\right)-m^{4} \bar{\alpha}_{1} \bar{\alpha}_{2} e^{2 k\left(X_{1}-X_{2}\right)}} d l
\end{aligned}
$$

for $X_{1}<X_{2}<x$, with $k=\sqrt{m^{2}+l^{2}}$. Between the lines, we find

$$
\begin{aligned}
& n_{+}^{*}(x)=n_{+}^{0} \\
&-\int_{-\infty}^{\infty} \frac{m^{4}}{4 \pi k} \frac{\bar{\alpha}_{1}\left(2 k+m^{2} \bar{\alpha}_{2}\right) e^{2 k\left(X_{1}-x\right)}+\bar{\alpha}_{2}\left(2 k+m^{2} \bar{\alpha}_{1}\right) e^{2 k\left(x-X_{2}\right)}-2 m^{2} \bar{\alpha}_{1} \bar{\alpha}_{2} e^{2 k\left(X_{1}-X_{2}\right)}}{\left(2 k+m^{2} \bar{\alpha}_{1}\right)\left(2 k+m^{2} \bar{\alpha}_{2}\right)-m^{4} \bar{\alpha}_{1} \bar{\alpha}_{2} e^{2 k\left(X_{1}-X_{2}\right)}} d l \\
& n_{-}^{*}(x)=n_{-}^{0} \\
&+\int_{-\infty}^{\infty} \frac{m^{2}}{4 \pi k} \frac{\bar{\alpha}_{1}(k+l)^{2}\left(2 k+m^{2} \bar{\alpha}_{2}\right) e^{2 k\left(X_{1}-x\right)}+\bar{\alpha}_{2}(k-l)^{2}\left(2 k+m^{2} \bar{\alpha}_{1}\right) e^{2 k\left(x-X_{2}\right)}+2 m^{4} \bar{\alpha}_{1} \bar{\alpha}_{2} e^{2 k\left(X_{1}-X_{2}\right)}}{\left(2 k+m^{2} \bar{\alpha}_{1}\right)\left(2 k+m^{2} \bar{\alpha}_{2}\right)-m^{4} \bar{\alpha}_{1} \bar{\alpha}_{2} e^{2 k\left(X_{1}-X_{2}\right)}} d l \\
& \rho^{*}(x)=-\int_{-\infty}^{+\infty} \frac{m^{2} k}{2 \pi} \frac{\bar{\alpha}_{1}\left(2 k+m^{2} \bar{\alpha}_{2}\right) e^{2 k\left(X_{1}-x\right)}+\bar{\alpha}_{2}\left(2 k+m^{2} \bar{\alpha}_{1}\right) e^{2 k\left(x-X_{2}\right)}}{\left(2 k+m^{2} \bar{\alpha}_{1}\right)\left(2 k+m^{2} \bar{\alpha}_{2}\right)-m^{4} \bar{\alpha}_{1} \bar{\alpha}_{2} e^{2 k\left(X_{1}-X_{2}\right)}} d l
\end{aligned}
$$


for $X_{1}<x<X_{2}$. It is straightforward to verify that these expressions reduce to case of one adsorbing line when $X_{1} \rightarrow-\infty$, as it should. Also, one can verify that for $X_{1}=X_{2}$, we recover the density profiles for one line with adhesivity $\bar{\alpha}_{1}+\bar{\alpha}_{2}$. Figure 15 shows the charge density profile in the presence of two positive lines.

The linear density of adsorbed particles by each line, $\sigma_{1}$ and $\sigma_{2}$, are obtained from $\sigma_{1}=\overline{\alpha_{1}} n_{+}^{*}\left(X_{1}\right)$ and a similar expression for $\sigma_{2}$. Explicitly,

$$
\begin{aligned}
\sigma_{1} & =\bar{\alpha}_{1} n_{+}^{0}-\frac{m^{4} \bar{\alpha}_{1}}{4 \pi} \int_{-\infty}^{+\infty} \frac{\left[\bar{\alpha}_{1}\left(2 k+m^{2} \bar{\alpha}_{2}\right)+\bar{\alpha}_{2}\left(2 k-m^{2} \bar{\alpha}_{1}\right) e^{2 k\left(X_{1}-X_{2}\right)}\right] d l}{k\left[\left(2 k+m^{2} \bar{\alpha}_{1}\right)\left(2 k+m^{2} \bar{\alpha}_{2}\right)-m^{4} \bar{\alpha}_{1} \bar{\alpha}_{2} e^{2 k\left(X_{1}-X_{2}\right)}\right]} \\
& =\frac{m^{4}}{2 \pi} \int_{-\infty}^{+\infty} \frac{\left(2 k+m^{2} \bar{\alpha}_{2}\right) \bar{\alpha}_{1}-m^{2} \bar{\alpha}_{1} \bar{\alpha}_{2} e^{2 k\left(X_{1}-X_{2}\right)}}{\left(2 k+m^{2} \bar{\alpha}_{1}\right)\left(2 k+m^{2} \bar{\alpha}_{2}\right)-m^{4} \bar{\alpha}_{1} \bar{\alpha}_{2} e^{2 k\left(X_{1}-X_{2}\right)}} d l
\end{aligned}
$$

and a similar expression for $\sigma_{2}$ interchanging $\bar{\alpha}_{1}$ and $\bar{\alpha}_{2}$. In the second line of Eq. (4.23) we used the formal expression

$$
n_{+}^{0}=\frac{m^{2}}{4 \pi} \int_{-\infty}^{+\infty} \frac{d l}{k}
$$

for the bulk density (actually, the integral should be cutoff to a $l_{\max }=e^{-C} / a$ to obtain finite results). The total adsorbed charge is found to be

$$
\sigma_{1}+\sigma_{2}=\frac{m^{2}}{\pi} \int_{-\infty}^{+\infty} \frac{\left(\bar{\alpha}_{1}+\bar{\alpha}_{2}\right) k+m^{2} \bar{\alpha}_{1} \bar{\alpha}_{2}\left[1-e^{2 k\left(X_{1}-X_{2}\right)}\right]}{\left(2 k+m^{2} \bar{\alpha}_{1}\right)\left(2 k+m^{2} \bar{\alpha}_{2}\right)-m^{4} \bar{\alpha}_{1} \bar{\alpha}_{2} e^{2 k\left(X_{1}-X_{2}\right)}}
$$

It is straightforward to verify that

$$
\sigma_{1}+\sigma_{2}=-\int_{-\infty}^{+\infty} \rho^{*}(x) d x
$$

as it should be, since the system is globally neutral.

In the case where line at $X_{2}$ adsorbs negative particles while the line at $X_{1}$ adsorbs the positive ones, we find the following density profiles

$$
\begin{aligned}
n_{+}(x) & =\left[1+\bar{\alpha}_{1} \delta\left(x-X_{1}\right)\right] n_{+}^{*}(x) \\
n_{-}(x) & =\left[1+\bar{\alpha}_{2} \delta\left(x-X_{2}\right)\right] n_{-}^{*}(x) \\
\rho(x) & =\bar{\alpha}_{1} \delta\left(x-X_{1}\right) n_{+}^{*}\left(X_{1}\right)-\bar{\alpha}_{2} \delta\left(x-X_{2}\right) n_{-}^{*}\left(X_{2}\right)+\rho^{*}(x)
\end{aligned}
$$

with

$$
\begin{array}{r}
n_{+}^{*}(x)=n_{+}^{0}-\int_{-\infty}^{\infty} \frac{m^{2} e^{-2 k x}}{4 \pi k} \frac{m^{2} \bar{\alpha}_{1}\left(2 k-m^{2} \bar{\alpha}_{2}\right) e^{2 k X_{1}}-\bar{\alpha}_{2}(k-l)^{2}\left(2 k+m^{2} \bar{\alpha}_{1}\right) e^{2 k X_{2}}}{\left(2 k+m^{2} \bar{\alpha}_{1}\right)\left(2 k+m^{2} \bar{\alpha}_{2}\right)+m^{2} \bar{\alpha}_{1} \bar{\alpha}_{2}(k+l)^{2} e^{2 k\left(X_{1}-X_{2}\right)}} d l \\
n_{-}^{*}(x)=n_{-}^{0}-\int_{-\infty}^{\infty} \frac{m^{2} e^{-2 k x}}{4 \pi k} \frac{\bar{\alpha}_{1}(k+l)^{2}\left(m^{2} \bar{\alpha}_{2}-2 k\right) e^{2 k X_{1}}+m^{2} \bar{\alpha}_{2}\left(2 k+m^{2} \bar{\alpha}_{1}\right) e^{2 k X_{2}}}{\left(2 k+m^{2} \bar{\alpha}_{1}\right)\left(2 k+m^{2} \bar{\alpha}_{2}\right)+m^{2} \bar{\alpha}_{1} \bar{\alpha}_{2}(k+l)^{2} e^{2 k\left(X_{1}-X_{2}\right)}} d l \\
\rho^{*}(x)=-\int_{-\infty}^{+\infty} \frac{m^{2} e^{-2 k x}}{2 \pi} \frac{\bar{\alpha}_{1}(k+l)\left(2 k-m^{2} \bar{\alpha}_{2}\right) e^{2 k X_{1}}+\bar{\alpha}_{2}(l-k)\left(2 k+m^{2} \bar{\alpha}_{1}\right) e^{2 k X_{2}}}{\left(2 k+m^{2} \bar{\alpha}_{1}\right)\left(2 k+m^{2} \bar{\alpha}_{2}\right)+m^{2}(k+l)^{2} \bar{\alpha}_{1} \bar{\alpha}_{2} e^{2 k\left(X_{1}-X_{2}\right)}} d l
\end{array}
$$




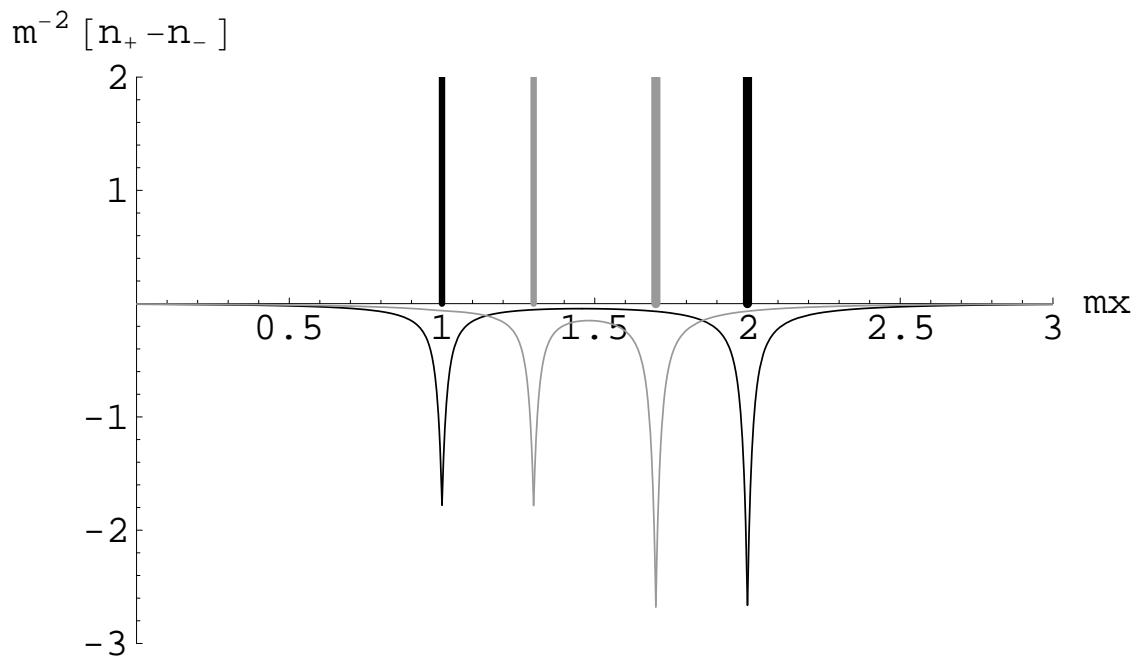

Figure 15: Charge density profile as a function of the distance. The adhesivities are $m \bar{\alpha}_{1}=0.2$ and $m \bar{\alpha}_{2}=0.3$. The position of the lines are $m X_{1}=1, m X_{1}=$ 1.3 and $m X_{2}=2, m X_{2}=1.7$ from the darkest to the lightest. The cutoff is $m a=0.01$.

for $X_{1}<X_{2}<x$. For $x$ between the lines, $X_{1}<x<X_{2}$, we find

$$
\begin{gathered}
n_{+}^{*}(x)=n_{+}^{0} \\
+\int_{-\infty}^{\infty} \frac{m^{2}}{4 \pi k} \frac{m^{2} \bar{\alpha}_{1}\left(-2 k-m^{2} \bar{\alpha}_{2}\right) e^{2 k\left(X_{1}-x\right)}+\bar{\alpha}_{2}(k+l)^{2}\left(2 k+m^{2} \bar{\alpha}_{1}\right) e^{2 k\left(x-X_{2}\right)}-2 m^{2} \bar{\alpha}_{1} \bar{\alpha}_{2}(k+l)^{2} e^{2 k\left(X_{1}-X_{2}\right)}}{\left(2 k+m^{2} \bar{\alpha}_{1}\right)\left(2 k+m^{2} \bar{\alpha}_{2}\right)+m^{2}(k+l)^{2} \bar{\alpha}_{1} \bar{\alpha}_{2} e^{2 k\left(X_{1}-X_{2}\right)}} d l \\
n_{-}^{*}(x)=n_{-}^{0} \\
+\int_{-\infty}^{\infty} \frac{m^{2}}{4 \pi k} \frac{\bar{\alpha}_{1}(k+l)^{2}\left(2 k+m^{2} \bar{\alpha}_{2}\right) e^{2 k\left(X_{1}-x\right)}-m^{2} \bar{\alpha}_{2}\left(2 k+m^{2} \bar{\alpha}_{1}\right) e^{2 k\left(x-X_{2}\right)}-2 m^{2} \bar{\alpha}_{1} \bar{\alpha}_{2}(k+l)^{2} e^{2 k\left(X_{1}-X_{2}\right)}}{\left(2 k+m^{2} \bar{\alpha}_{1}\right)\left(2 k+m^{2} \bar{\alpha}_{2}\right)+m^{2}(k+l)^{2} \bar{\alpha}_{1} \bar{\alpha}_{2} e^{2 k\left(X_{1}-X_{2}\right)}} d l \\
\rho^{*}(x)=-\int_{-\infty}^{+\infty} \frac{m^{2}(k+l)}{2 \pi} \frac{\bar{\alpha}_{1}\left(2 k+m^{2} \bar{\alpha}_{2}\right) e^{2 k\left(X_{1}-x\right)}-\bar{\alpha}_{2}\left(2 k+m^{2} \bar{\alpha}_{1}\right) e^{2 k\left(x-X_{2}\right)}}{\left(2 k+m^{2} \bar{\alpha}_{1}\right)\left(2 k+m^{2} \bar{\alpha}_{2}\right)+m^{2}(k+l)^{2} \bar{\alpha}_{1} \bar{\alpha}_{2} e^{2 k\left(X_{1}-X_{2}\right)}} d l
\end{gathered}
$$

The linear charge density adsorbed by each line is now given by $\sigma_{1}=$ 


$$
\begin{aligned}
& \bar{\alpha}_{1} n_{+}^{*}\left(X_{1}\right) \text { and } \sigma_{2}=-\bar{\alpha}_{2} n_{-}^{*}\left(X_{2}\right), \\
& \sigma_{1}=\frac{\alpha_{1} m^{2}}{2 \pi} \int_{-\infty}^{+\infty} \frac{\left[2 k+m^{2} \bar{\alpha}_{2}+(k+l)^{2} \bar{\alpha}_{2} e^{2 k\left(X_{1}-X_{2}\right)}\right] d l}{\left(2 k+m^{2} \bar{\alpha}_{1}\right)\left(2 k+m^{2} \bar{\alpha}_{2}\right)+m^{2}(k+l)^{2} \bar{\alpha}_{1} \bar{\alpha}_{2} e^{2 k\left(X_{1}-X_{2}\right)}} \\
& \sigma_{2}=-\frac{\alpha_{2} m^{2}}{2 \pi} \int_{-\infty}^{+\infty} \frac{\left[2 k+m^{2} \bar{\alpha}_{1}+(k+l)^{2} \bar{\alpha}_{1} e^{2 k\left(X_{1}-X_{2}\right)}\right] d l}{\left(2 k+m^{2} \bar{\alpha}_{1}\right)\left(2 k+m^{2} \bar{\alpha}_{2}\right)+m^{2}(k+l)^{2} \bar{\alpha}_{1} \bar{\alpha}_{2} e^{2 k\left(X_{1}-X_{2}\right)}}
\end{aligned}
$$

The total linear charge density adsorbed by the lines is formally given by

$\sigma_{1}+\sigma_{2}=\left(\bar{\alpha}_{1}-\bar{\alpha}_{2}\right) \frac{m^{2}}{\pi} \int_{-\infty}^{+\infty} \frac{k d l}{\left(2 k+m^{2} \bar{\alpha}_{1}\right)\left(2 k+m^{2} \bar{\alpha}_{2}\right)+m^{2}(k+l)^{2} \bar{\alpha}_{1} \bar{\alpha}_{2} e^{2 k\left(X_{1}-X_{2}\right)}}$

Once again, it is straightforward to verify that

$$
\sigma_{1}+\sigma_{2}=-\int_{-\infty}^{+\infty} \rho^{*}(x) d x
$$

as imposed by the global neutrality of the system.

It is interesting to notice that in the two cases considered in this section, the density profile of particles that are adsorbed by one line is a continuous function as it crosses the line, while the density profile of the particles that are not adsorbed by this line is discontinuous when crossing such line. However, the discontinuity jump in this density is exponentially small for large separations between the two lines, it is of order $e^{-2 m\left|X_{1}-X_{2}\right|}$.

\subsection{Grand potential}

In the following subsections, we compute the grand potential of the plasma with one or two adsorbing lines at $\Gamma=2$, by solving the eigenvalue problem (2.6).

\subsubsection{An infinite line}

For this case we confine the plasma into a rectangular box of area $2 L_{x} \times L_{y}$ and we work in Cartesian coordinates. The boundaries and the adsorbing line, located at $x=0$, divide the space in four regions $x<-L_{x},-L_{x}<x<0$, $0<x<L_{x}$ and $x>L_{x}$ which will be labeled by (1), (2), (3) and (4).

The eigenvalue system (2.6) takes the form

$$
\begin{aligned}
m\left[1+\bar{\alpha}_{1} \delta(x)\right] g(\mathbf{r}) & =2 \lambda \partial_{z} f(\mathbf{r}), \\
m f(\mathbf{r}) & =2 \lambda \partial_{\bar{z}} g(\mathbf{r}) .
\end{aligned}
$$

for $-L_{x} \leq x \leq L_{x}$. Outside that region, $m_{+}(x)=m_{-}(x)=0$. Thus we conclude that for $x \notin\left[-L_{x}, L_{x}\right], \partial_{z} f=0$ and $\partial_{\bar{z}} g=0$, that is $f$ is an antianalytic function of $z$ and $g$ is an analytic function of $z$. 
The translation symmetry on the $y$ axis allows us to assume $g(\mathbf{r})$ of the form $g(x, y)=\tilde{g}(x) e^{i l y}$. The general solution for the four regions can be written as

$$
\begin{aligned}
& g^{(1)}(x, y)=A^{(1)} e^{l x+i l y} \\
& g^{(2)}(x, y)=A^{(2)} e^{k_{x} x+i l y}+B^{(2)} e^{-\left(k_{x} x-i l y\right)} \\
& g^{(3)}(x, y)=A^{(3)} e^{k_{x} x+i l y}+B^{(3)} e^{-\left(k_{x} x-l y\right)} \\
& g^{(4)}(x, y)=B^{(4)} e^{l x+i l y}
\end{aligned}
$$

and

$$
\begin{aligned}
f^{(1,4)}(x, y) & =C^{(1,4)} e^{-l x+i l y} \\
f^{(2,3)}(x, y) & =\frac{\lambda}{m}\left(\frac{\partial}{\partial x}+i \frac{\partial}{\partial y}\right) g^{(2,3)}(x, y) .
\end{aligned}
$$

with $k_{x}=\sqrt{l^{2}+\frac{m^{2}}{\lambda^{2}}}$.

Since the eigenfunctions must vanish at $x= \pm \infty$, we conclude that $g^{(1)}=$ $f^{(4)}=0$ for $l<0$ and $f^{(1)}=g^{(4)}=0$ if $l>0$. The boundary conditions demand that both $g(x, y)$ and $f(x, y)$ must be continuous at $x= \pm L_{x}$. At $x=0$, $g(x, y)$ is continuous and $f(x, y)$ is discontinuous due to the Dirac distribution in Eq. (4.33a):

$$
f\left(0^{+}, y\right)-f\left(0^{-}, y\right)=\frac{m \bar{\alpha}_{1}}{\lambda} g(0, y) .
$$

Assuming $l>0$, these boundary conditions can be expressed by the linear system

$$
\left(\begin{array}{cccc}
\left(k_{x}-l\right) e^{-k_{x} L_{x}} & -\left(k_{x}+l\right) e^{k_{x} L_{x}} & 0 & 0 \\
0 & 0 & e^{k_{x} L_{x}} & e^{-k_{x} L_{x}} \\
1 & 1 & -1 & -1 \\
m^{2} \bar{\alpha}_{1}-\lambda^{2}\left(k_{x}-l\right) & m^{2} \bar{\alpha}_{1}+\lambda^{2}\left(k_{x}+l\right) & \lambda^{2}\left(k_{x}-l\right) & -\lambda^{2}\left(k_{x}+l\right)
\end{array}\right)\left(\begin{array}{c}
A_{2} \\
B_{2} \\
A_{3} \\
B_{3}
\end{array}\right)=0 .
$$

The functions $g(x, y)$ and $f(x, y)$ must not be zero, hence we demand that the determinant of the latter matrix must vanish. This condition leads us to the relation

$\cosh \left(2 k_{x} L_{x}\right)\left[1+\frac{m^{2} \bar{\alpha}_{1} l \lambda^{-2}}{2 k_{x}^{2}}\right]+\sinh \left(2 k_{x} L_{x}\right)\left[\frac{l}{k_{x}}+\frac{m^{2} \bar{\alpha}_{1} \lambda^{-2}}{2 k_{x}}\right]+\frac{m^{2} \bar{\alpha}_{1} l \lambda^{-2}}{2 k_{x}^{2}}=0$.

For each value of $l$, there are several possible solutions to Eq. (4.38) for the eigenvalue $\lambda$, which we will denote as $\left\{\lambda_{l, n}\right\}_{n}$. For $l<0$, we obtain (4.38) changing $l$ by $-l$. To obtain the grand potential, we can recognize its relationship with a Weierstrass product [18] as follows. Let us define the analytic function, for $l \geq 0$

$$
\begin{aligned}
h_{l}(z) & =\left(1+\frac{m^{2} \bar{\alpha}_{1} l z^{2}}{2\left(m^{2} z^{2}+l^{2}\right)}\right) \cosh \left(2 \sqrt{m^{2} z^{2}+l^{2}} L_{x}\right)+\frac{m^{2} \bar{\alpha}_{1} l z^{2}}{2\left(m^{2} z^{2}+l^{2}\right)} \\
& +\left(\frac{l}{\sqrt{l^{2}+m^{2} z^{2}}}\right)\left(1+\frac{m^{2} \bar{\alpha}_{1} z^{2}}{2 l}\right) \sinh \left(2 \sqrt{m^{2} z^{2}+l^{2}} L_{x}\right) .
\end{aligned}
$$


We have $h_{l}(0)=e^{2 l L_{x}}, h_{l}^{\prime}(0)=0$ and $h_{l}(z)=h_{l}(-z)$. By construction, the zeros of $h_{l}$ are precisely the inverse of the eigenvalues $1 / \lambda_{l, n}$. The representation of $h_{l}(z)$ as a Weierstrass infinite product is therefore

$$
h_{l}(z)=h_{l}(0) \prod_{n}\left(1-z \lambda_{l, n}\right)
$$

Then, we notice that the grand potential is simply

$$
\beta \Omega=-\sum_{l} \ln \prod_{n}\left(1+\lambda_{l, n}\right)=-\frac{L_{y}}{\pi} \int_{0}^{\infty} d l \ln \left[e^{-2 l L_{x}} h_{l}(-1)\right]
$$

where we replaced the sum over $l$ by an integral in the thermodynamic limit $L_{y} \rightarrow \infty$. In the thermodynamic limit, we also consider that $L_{x} \rightarrow \infty$, and we approximate $\cosh \left(2 k L_{x}\right) \sim \sinh \left(2 k L_{x}\right) \sim e^{2 k L_{x}} / 2$, with $k=\sqrt{l^{2}+m^{2}}$. The final result is

$$
\beta \Omega=\beta \Omega_{0}+\beta \Omega_{\mathrm{exc}}\left(\bar{\alpha}_{1}\right)
$$

with

$$
\beta \Omega_{\mathrm{exc}}\left(\bar{\alpha}_{1}\right)=-\frac{L_{y}}{\pi} \int_{0}^{+\infty} \ln \left[1+\frac{m^{2} \bar{\alpha}_{1}}{2 \sqrt{m^{2}+l^{2}}}\right] d l
$$

and $\Omega_{0}$ the grand potential of the unperturbed system for this geometry,

$$
\Omega_{0}=-2 L_{x} L_{y} p_{b}+2 L_{y} \gamma+\mathcal{O}\left(e^{-2 m L_{x}}\right)
$$

with the bulk pressure $p_{b}$ and the surface tension near an impenetrable wall $\gamma$,

$$
\begin{aligned}
\beta p_{b} & =\frac{m^{2}}{2 \pi}\left[\ln \frac{2}{m a}-C+\frac{1}{2}\right]=n_{0}+\frac{m^{2}}{4 \pi}, \\
\beta \gamma & =m\left[\frac{1}{4}-\frac{1}{2 \pi}\right] .
\end{aligned}
$$

Hence we can conclude that the effective chemical potential of a line of length $L_{y}$ and adhesivity $\bar{\alpha}$ is $\mu(\bar{\alpha})=\Omega_{\text {exc }}^{ \pm}(\bar{\alpha})$. The integral (4.43) can be computed explicitly. Actually, one must introduce an ultraviolet cutoff $l_{\max }=e^{-C} / a$ to obtain a finite result. This choice of the cutoff is done to ensure that the result for bulk pressure (4.45) is the same as the one obtained by integrating the bulk densities (3.7) [1]. Neglecting terms that vanish when $a \rightarrow 0$, we have

$$
\beta \Omega_{\mathrm{exc}}(\bar{\alpha})=-\frac{m L_{y}}{\pi}\left[\tilde{\alpha} \ln \frac{2 e^{-C}}{m a}-\sqrt{\tilde{\alpha}^{2}-1} \operatorname{arctanh} \frac{\sqrt{\tilde{\alpha}^{2}-1}}{\tilde{\alpha}}-\frac{\pi}{2}+\tilde{\alpha}\right]
$$

with $\tilde{\alpha}=m \bar{\alpha} / 2$. It is interesting to compare this result with the surface tension obtained in a similar problem where the plasma is confined in a strip with adsorbing boundaries [10]. In that problem the adsorbing line was located at a 
hard wall boundary. In our notations, the contribution of both the hard wall and the adsorbing line was [10]

$$
\beta \Omega_{\mathrm{exc}}^{\text {adsorbing wall }}=-\frac{m L_{y}}{4 \pi}\left[m \bar{\alpha} \ln \frac{2 e^{-C}}{m a}+1-\pi+m \bar{\alpha}+\frac{1-(m \bar{\alpha})^{2}}{\bar{\alpha}} \ln (1+m \bar{\alpha})\right]
$$

We notice that $\Omega_{\text {exc }}(\bar{\alpha})+L_{y} \gamma$ of our present problem is different from $\Omega_{\text {exc }}^{\text {adsorbing wall }}$. This is to be expected since each situation is different. In our present problem the adsorbing line is very far from the hard wall boundaries, while in Ref. [10], the adsorbing line is on the boundary itself. We notice however that the (divergent) dominant term, when the cutoff $a$ vanish, for our problem is twice the one for the adsorbing boundary

$$
\Omega_{\text {exc }}(\bar{\alpha}) \sim 2 \Omega_{\text {exc }}^{\text {adsorbing wall }} \sim \frac{m L_{y}}{2 \pi} \ln (m a) .
$$

The factor 2 between both expressions can be understood if we realize that in our present problem the plasma is on both sides of the adsorbing line, while in Ref. [10] it is only on one side, the other side is empty.

From Eqs. (4.43) and (4.11a) or from Eqs. (4.47) and (4.11b), one can check that the relation between the adsorbed charge density $\sigma$ and the excess grand potential

$$
\sigma=-\bar{\alpha}_{1} \frac{\partial\left(\beta \Omega / L_{y}\right)}{\partial \bar{\alpha}_{1}}
$$

is satisfied.

\subsubsection{Two infinite lines}

Let us now consider the case where there are two infinite parallel adsorbing lines which attract the same kind of particles, say positive. The eigenvalue problem which must be solved is

$$
\begin{aligned}
m f(\mathbf{r}) & =2 \lambda \partial_{\bar{z}} g(\mathbf{r}) \\
m\left[1+\bar{\alpha}_{1} \delta\left(x-X_{1}\right)+\bar{\alpha}_{2}\left(x-X_{2}\right)\right] g(\mathbf{r}) & =2 \lambda \partial_{z} f(\mathbf{r}) .
\end{aligned}
$$

We have placed the first line at $x=X_{1}$ and the second one at $x=X_{2}$. The method of solution is similar to the one for one line. Following similar steps as in the previous section, we find after some algebra [13]

$$
\beta \Omega=\beta \Omega_{0}+\beta \Omega_{\mathrm{exc}}\left(\bar{\alpha}_{1}\right)+\beta \Omega_{\mathrm{exc}}\left(\bar{\alpha}_{2}\right)+\beta \Omega^{ \pm \pm}
$$

with

$$
\beta \Omega^{ \pm \pm}=-\frac{L_{y}}{\pi} \int_{0}^{+\infty} \ln \left[1-\frac{m^{4} \bar{\alpha}_{1} \bar{\alpha}_{2} e^{-2 k\left|X_{1}-X_{2}\right|}}{\left(2 k+m^{2} \bar{\alpha}_{1}\right)\left(2 k+m^{2} \bar{\alpha}_{2}\right)}\right] d l
$$

and $\beta \Omega_{\mathrm{exc}}(\bar{\alpha})$ given in (4.43). We recall that $k=\sqrt{m^{2}+l^{2}}$. 


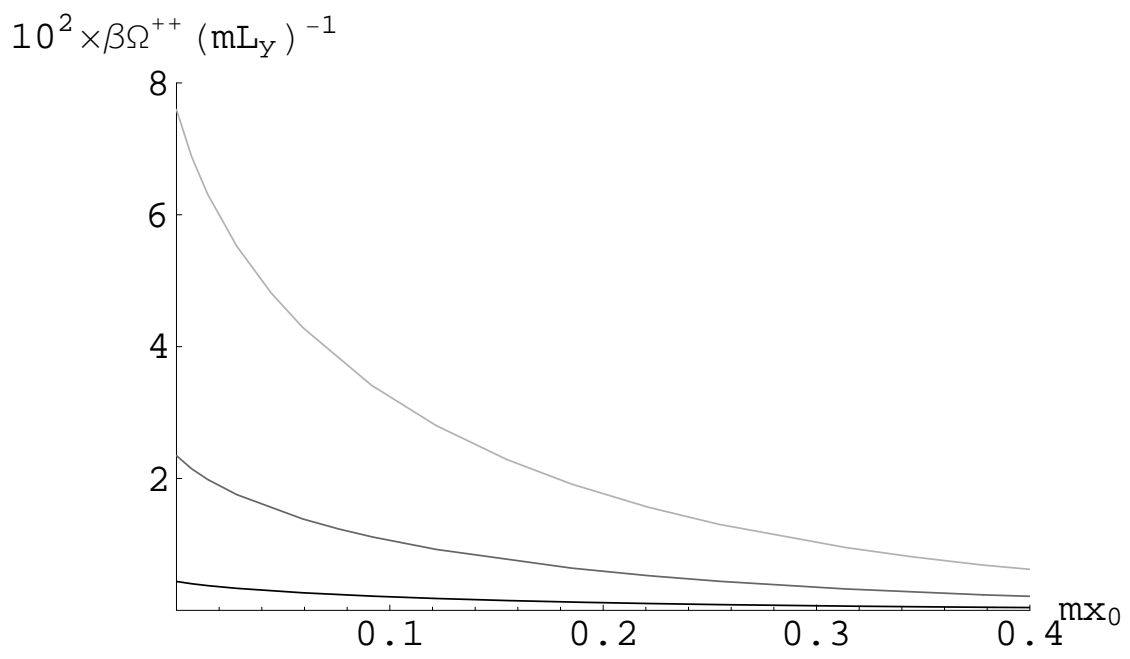

Figure 16: Effective repulsion between two "positive" lines as a function of their distance $2 x_{0}=\left|X_{1}-X_{2}\right|$. The adhesivities are $m \bar{\alpha}_{1}=m \bar{\alpha}_{2}=0.2,0.5,1$ from the darkest to the lightest. The cutoff is $m a=0.01$.

When the lines attract different types of particles the grand potential is

$$
\beta \Omega=\beta \Omega_{0}+\beta \Omega_{\mathrm{exc}}\left(\bar{\alpha}_{1}\right)+\beta \Omega_{\mathrm{exc}}\left(\bar{\alpha}_{2}\right)+\beta \Omega^{ \pm \mp}
$$

with

$$
\beta \Omega^{ \pm \mp}=-\frac{L_{y}}{2 \pi} \int_{-\infty}^{+\infty} \ln \left[1+\frac{m^{2} \bar{\alpha}_{1} \bar{\alpha}_{2}(k+l)^{2} e^{-2 k\left|X_{1}-X_{2}\right|}}{\left(2 k+m^{2} \bar{\alpha}_{1}\right)\left(2 k+m^{2} \bar{\alpha}_{2}\right)}\right] d l .
$$

The effective interaction between two lines (depending on their sign) is given by the expressions (4.53) and (4.55).

Figures 16 and 17 show the effective interaction between two infinite lines. As expected, like in the problem for point impurities, there is a repulsion if the sign of the lines is equal and a repulsion if it is different.

Notice that in both cases, $s= \pm, s^{\prime}= \pm$, in the expression of the full excess grand potential $\Omega_{\text {exc }}\left(\bar{\alpha}_{1}\right)+\Omega_{\text {exc }}\left(\bar{\alpha}_{2}\right)+\Omega^{s s^{\prime}}$ appears the logarithm of a term which is precisely the denominator in the Green functions and the density profiles Eqs. (4.21), (4.22), (4.28) and (4.29). Also, one can easily check that the adsorbed charge on each line, computed in Eqs. (4.23) and (4.30), can also be obtained from

$$
\sigma_{i}=-\frac{\beta}{L_{y}} \alpha_{i} \frac{\partial}{\partial \alpha_{i}}\left(\Omega_{\mathrm{exc}}\left(\bar{\alpha}_{1}\right)+\Omega_{\mathrm{exc}}\left(\bar{\alpha}_{2}\right)+\Omega^{s s^{\prime}}\right)
$$

for $i=1,2$. 


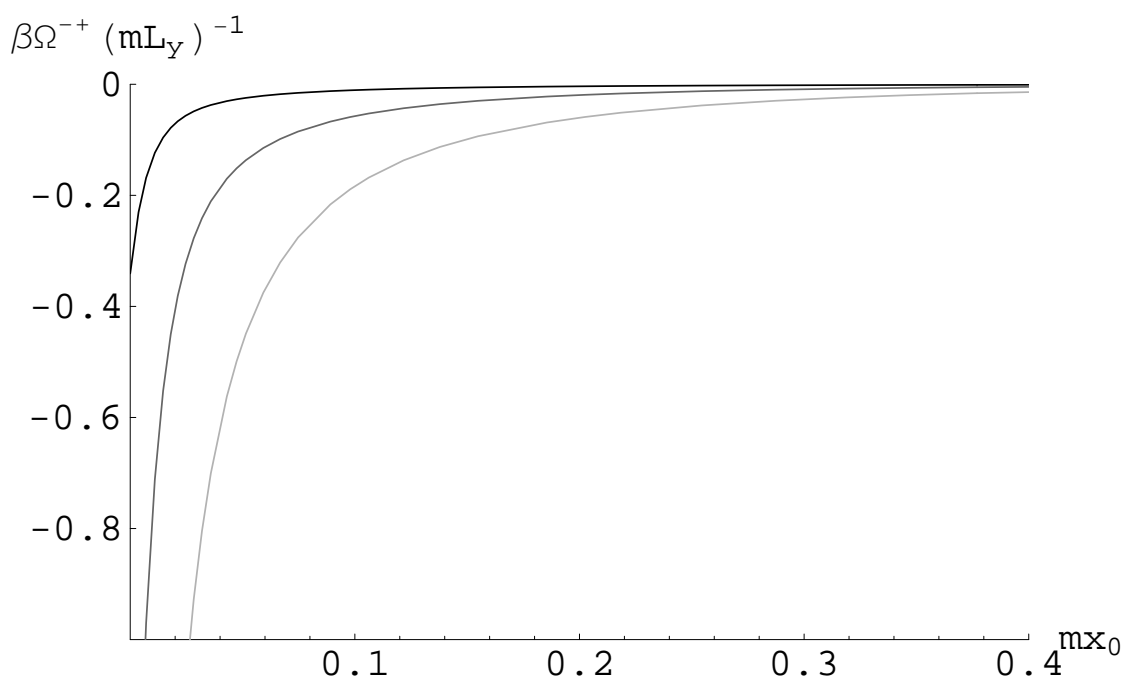

Figure 17: Effective attraction between a positive and a negative line as a function of their distance $2 x_{0}=\left|X_{1}-X_{2}\right|$. The values for the different parameters are the same as in figure 16 .

\section{Summary}

In the present document we analyzed the behavior of the two-component plasma at $\Gamma=2$ in the presence of one and two point adsorbing impurities, or in the presence of one and two adsorbing lines.

For point impurities, as shown in Ref. [8], the partition function and correlations of the system can be expressed in terms of the same quantities for an unperturbed system, without impurities. Since at $\Gamma=2$, exact results are available for the partition function and correlations of the unperturbed system, we were able to obtain exact results for the partition function, the density profiles and correlation functions of the plasma with one or two point impurities. We also computed the electric potential created by one impurity, due to the charge it adsorbs and the polarization cloud that is formed around it.

As a simple model for electrodes with adsorbing sites, we studied the properties of the plasma with one or two parallel lines of absorbing impurities. The general formalism developed in Ref. [1] for the two-component plasma with an external potential is applied to this case. We obtained exact results for the partition function and density profiles of the plasma. In both cases we checked that various relations between the adsorbed charge and the excess grand potential are satisfied. 


\section{Acknowledgments}

The authors acknowledge partial financial support from Comité de Investigaciones de la Facultad de Ciencias de la Universidad de los Andes and from ECOS Nord/COLCIENCIAS.

\section{References}

[1] F. Cornu and B. Jancovici, The electrical double layer: A solvable model, J. Chem. Phys. 90:2444 (1989).

[2] L. Šamaj and I. Travěnec, Thermodynamic properties of the twodimensional two-component plasma, J. Stat. Phys. 101:713 (2000).

[3] L. Šamaj and B. Jancovici, Surface tension of a metal - electrolyte boundary: exactly solvable model, J. Stat. Phys. 103:717 (2001).

[4] L. Šamaj, Surface tension of an ideal dielectric - electrolyte boundary: exactly solvable model, J. Stat. Phys. 103:737 (2001).

[5] B. Jancovici and G. Manificat, Classical Coulomb fluids in a confined geometry, J. Stat. Phys. 68:1089 (1992).

[6] B. Jancovici and G. Téllez, Coulomb systems seen as critical systems: Ideal conductor boundaries, J. Stat. Phys. 82:609 (1996).

[7] G. Téllez, Two-component plasma in a gravitational field, J. Chem. Phys. 106:8572 (1997).

[8] M. L. Rosinberg, J. L. Lebowitz, L. Blum, Solvable model for localized adsorption in a Coulomb system, J. Stat. Phys. 44:153 (1986).

[9] F. Cornu, Two-dimensional models for an electrode with adsorption sites, J. Stat. Phys. 54:681 (1989).

[10] G. Téllez, L. Merchán, Solvable model for electrolytic soap films: the twodimensional two-component plasma, J. Stat. Phys. 108:495 (2002).

[11] L. Merchán, G. Téllez, Confined Coulomb systems with adsorbing boundaries: the two-dimensional two-component plasma, J. Stat. Phys. 114:735 (2004).

[12] M. Abramowitz and I. A. Stegun, Handbook of Mathematical Functions (National Bureau of Standards, 1964).

[13] A. Ferrero, Analytic solution to the two-component plasma with impurities, Master Thesis, Universidad de los Andes (2006).

[14] B. Jancovici, Classical Coulomb systems near a plane wall. I, J. Stat. Phys. 28:43 (1982). 
[15] B. Jancovici, Classical Coulomb systems near a plane wall. II, J. Stat. Phys. 29:263 (1982).

[16] Ph. A. Martin, Sum rules in charged fluid, Rev. Mod. Phys. 60:1075 (1988).

[17] G. Téllez, Short-distance expansion of correlation functions for the charge-symmetric two-dimensional two-component plasma: exact results, J. Stat. Mech. P10001 (2005).

[18] E. T. Whittaker and G. N. Watson, A course on modern analysis (Cambridge University press, 1927) 